\title{
Chemo- and Regioselective Functionalization of Uracil Derivatives. Applications to the Synthesis of Oxypurinol and Emivirine
}

\author{
Nadège Boudet and Paul Knochel* \\ Department Chemie und Biochemie, Ludwig-Maximilians-Universität, \\ Butenandtstrasse 5-13, 81377, München (Germany). \\ Paul.Knochel@cup.uni-muenchen.de
}

\section{Supporting information}

Experimental procedures, analytical and spectroscopy data for starting materials and final products (45 pages). 


\section{General considerations}

Unless otherwise indicated, all reactions were carried out with magnetic stirring and, if air or moisture sensitive, in flame-dried glassware under argon. Syringes used to transfer reagents and solvent were purged with argon prior to use. Reactions were monitored by gas chromatography (GC and GC-MS) or thin layer chromatography (TLC).

\section{Preparation of the reagent $i$-PrMgCl$\cdot \mathbf{L i C l}$ :}

Magnesium turnings $(110 \mathrm{mmol})$ and anhydrous $\mathrm{LiCl}(100 \mathrm{mmol})$ were placed in an Arflushed flask and THF $(25 \mathrm{~mL})$ was added. A solution of $i$-PrCl $(100 \mathrm{mmol})$ in THF $(25 \mathrm{~mL})$ was slowly added at rt. The reaction starts within a few minutes. After addition, the reaction mixture was stirred for $12 \mathrm{~h}$ at $\mathrm{rt}$. The grey solution of $i-\mathrm{PrMgCl} \cdot \mathrm{LiCl}$ was cannulated to an other flask under Ar and removed in this way from excess of magnesium. A yield of ca. 95$98 \%$ of $i$-PrMgCl. $\mathrm{LiCl}$ is obtained.

Preparation of the reagent $\mathrm{CuCN} \cdot 2 \mathrm{LiCl}$ in $\mathrm{THF}^{\mathbf{1}}$ : according to the known procedure.

\section{General procedure for the $\mathrm{Br} / \mathrm{Mg}$ exchange reaction:}

A dry and argon flushed $10 \mathrm{~mL}$ flask, equipped with a magnetic stirring bar and a septum, was charged with the neat 5-bromo-4-halogeno-2,6-dimethoxypyrimidine (1 mmol) dissolved in dry THF (1.0 mL). $i$-PrMgCl. LiCl (1 mL, 1.05 M in THF, $1.05 \mathrm{mmol})$ was added slowly, dropwise, at appropriate temperature (as stated in the experiment). The reaction mixture was stirred at the same or plus $5^{\circ} \mathrm{C}$ temperature, and the completion of the $\mathrm{Br} / \mathrm{Mg}$ exchange was checked by GC-analysis using tetradecane as internal standard or by TLC.

\section{General procedure for the reaction with electrophiles:}

The freshly prepared magnesium reagent was cooled to the corresponding temperature or used at room temperature and the corresponding electrophile (1.1 mmol, 1.1 equiv.) was added. The mixture was stirred for time depending of the reactivity of the electrophile. The consumption of the magnesium reagent was checked by GC-analysis, using tetradecane as internal standard. After the reaction was completed, sat. $\mathrm{NH}_{4} \mathrm{Cl}$ solution was added and the mixture was extracted three times with $\mathrm{Et}_{2} \mathrm{O}$ or AcOEt. The solvent was evaporated and the product was purified by flash chromatography $\left(\mathrm{SiO}_{2}\right)$.

\footnotetext{
${ }^{1}$ Knochel, P.; Yeh, M. C. P.; Berk, S. C.; Talbert, J. J. Org. Chem. 1988, 53, 2390.
} 


\section{Starting materials:}

\section{5-bromo-4-chloro-2,6-dimethoxypyrimidine (4).}

This compound was prepared starting from 4-chloro-2,6-dimethoxypyrimidine according to the literature procedure. ${ }^{2}$ The analytic data correspond to the analytic data from the literature. The product 4 was obtained as a white solid (mp: 98,7-99, $7^{\circ} \mathrm{C}$ ).

${ }^{1} \mathrm{H}-\mathrm{NMR}\left(\mathrm{CDCl}_{3}, 300 \mathrm{MHz}\right): \delta=4.05$ (s, 3H), 3.99 (s, 3H).

${ }^{13} \mathrm{C}-\mathrm{NMR}\left(\mathrm{CDCl}_{3}, 75 \mathrm{MHz}\right): \delta=168.3,162.8,160.4,97.1,55.8$.

MS (EI, $70 \mathrm{ev),} \mathrm{m/z} \mathrm{( \% ):} 253.9$ (100), 252.9 (77), 251.9 (75), 250.9 (58), 238.9 (27), 223.9 (92), 221.0 (70), 208.9 (37), 143.0 (42).

HRMS (EI): calcd. for $\mathrm{C}_{6} \mathrm{H}_{6} \mathrm{BrClN}_{2} \mathrm{O}_{2}\left[\mathrm{M}^{+}\right]$: 251.9301, found: 251.9284 .

Starting material of 4,5-bromo-2,6-dimethoxypyrimidine (5): 4-bromo-2,6-dimethoxypyrimidine.

4-bromo-2,6-dimethoxypyrimidine was prepared starting from barbituric acid in two steps, according to the literature procedure ${ }^{3}$. The analytic data correspond to the analytic data from the literature.

The product was obtained as a white solid (mp: 90,9-92,0 ${ }^{\circ} \mathrm{C}$ ).

${ }^{1} \mathrm{H}-\mathrm{NMR}\left(\mathrm{CDCl}_{3}, 300 \mathrm{MHz}\right): \delta=6.52(\mathrm{~s}, 1 \mathrm{H}), 3.93$ (s, 3H), 3.89 (s, 3H).

${ }^{13} \mathrm{C}-\mathrm{NMR}\left(\mathrm{CDCl}_{3}, 75 \mathrm{MHz}\right): \delta=172.1,164.9,152.4,105.4,55.8,54.8$.

MS (EI, $70 \mathrm{ev),} \mathrm{m/z} \mathrm{( \% ):} 219.9$ (79), 218.9 (67), 217.9 (78), 216.9 (75), 189.9 (90), 187.9 (100), 174.9 (37), $109.0(51), 82.0$ (65).

IR (KBr): 1589 (m), 1550 (s), 1463 (s), 1363 (m), 1199 (m), 819 (s).

HRMS (EI): calcd. for $\mathrm{C}_{6} \mathrm{H}_{7} \mathrm{BrN}_{2} \mathrm{O}_{2}\left[\mathrm{M}^{+}\right]$: 217.9691, found: 217.9692 .

\section{5,4-dibromo -2,6-dimethoxypyrimidine (5).}

This compound (5) was prepared with the same procedure for the synthesis of 5-bromo-4chloro-2,6-dimethoxypyrimidine (4), starting from 4-bromo-2,6-dimethoxypyrimidine. 5,4dibromo-2,6-dimethoxypyrimidine (5) was isolated as a white solid in 96\% yield (mp: 117.6$\left.118.0{ }^{\circ} \mathrm{C}\right)$.

${ }^{1} \mathrm{H}-\mathrm{NMR}\left(\mathrm{CDCl}_{3}, 400 \mathrm{MHz}\right): \delta=4.05$ (s, 3H), 3.99 (s, 3H).

${ }^{13} \mathrm{C}-\mathrm{NMR}\left(\mathrm{CDCl}_{3}, 100 \mathrm{MHz}\right): \delta=167.7,162.54,153.74,100.80,55.78$.

${ }^{2}$ Okafor, C. J. Org. Chem. 1973, 38, 4386

${ }^{3}$ White, J. D.; Hansen, J. D. J. Org. Chem. 2005, 70, 1963-1977. 
MS (EI, $70 \mathrm{ev}), \mathrm{m} / z$ (\%): 299.8 (46), 298.8 (47), 297.8 (100), 296.8 (71), 295.8 (48), 294.8 (31), 282.8 (20), 269.8 (31), 267.8 (64), 265.8 (30), 188.9 (19), 186.9 (21).

IR (KBr): 2957 (w), 1526 (s), 1455 (m), 1343 (s), 1311 (m), 1008 (s).

HRMS (EI): calcd. for $\mathrm{C}_{6} \mathrm{H}_{6} \mathrm{Br}_{2} \mathrm{~N}_{2} \mathrm{O}_{2}\left[\mathrm{M}^{+}\right]$: 295.8796, found: 295.8799 .

\section{Products 8a-9e:}

\section{(4-chloro-2,6-dimethoxypyrimidin-5-yl)(phenyl)methanol (8a).}

A dry and argon flushed $10 \mathrm{~mL}$ flask, equipped with a magnetic stirring bar and a septum, was charged with a solution of 5-bromo-4-chloro-2,6-dimethoxypyrimidine (4) (254 mg, $1 \mathrm{mmol}$ ) in dry THF $(1 \mathrm{~mL}), i$-PrMgCl$\cdot \mathrm{LiCl}(1.0 \mathrm{M} / \mathrm{THF}, 1.05 \mathrm{mmol}, 1.05$ equiv) was added slowly at room temperature and the resulting mixture was stirred for 15 min to complete the brominemagnesium exchange (checked by GC-MS analysis of reaction aliquots). Benzaldehyde (117 $\mathrm{mg}, 1.1 \mathrm{mmol}, 1.1$ equiv) was added dropwise. The mixture was stirred for $5 \mathrm{~h}$ and was quenched with saturated aqueous $\mathrm{NH}_{4} \mathrm{Cl}$ solution. The aqueous phase was extracted with ether $(3 \times 5 \mathrm{~mL})$. The organic fractions were dried $\left(\mathrm{Na}_{2} \mathrm{SO}_{4}\right)$ and concentrated in vacuo. Purification by flash chromatography $(n$-pentane/diethyl ether $=3: 2)$ yielded $255 \mathrm{mg}(91 \%$ yield) of $\mathbf{3 a}$ as a white solid (mp.: $90,1-91,4^{\circ} \mathrm{C}$ ).

${ }^{1} \mathrm{H}-\mathrm{NMR}\left(\mathrm{CDCl}_{3}, 400 \mathrm{MHz}\right): \delta=7.33-7.24(\mathrm{~m}, 5 \mathrm{H}), 6.21(\mathrm{~d}, J=11 \mathrm{~Hz}, 1 \mathrm{H}), 4.01(\mathrm{~s}, 3 \mathrm{H})$, $3.96(\mathrm{~s}, 3 \mathrm{H}), 3.41(\mathrm{~d}, J=11 \mathrm{~Hz}, 1 \mathrm{H})$.

${ }^{13} \mathrm{C}-\mathrm{NMR}\left(\mathrm{CDCl}_{3}, 100 \mathrm{MHz}\right): 169.67,163.17,159.42,141.68,128.31,127.45,125.26$, 113.96, 69.98, 55.44, 55.00.

MS (EI, $70 \mathrm{ev),} \mathrm{m/z} \mathrm{( \% ):} 282.0$ (15), 280.0 (44), 262.0 (20), 205.0 (29), 203.0 (100), 105.0 (10), $77.0(12)$.

IR (KBr): 3412 (s), 2955 (w), 1583 (s), 1553 (s), 1464 (m), 1375(s), 1242 (m), 1032 (s).

HRMS (EI): calcd. for $\mathrm{C}_{13} \mathrm{H}_{13} \mathrm{ClN}_{2} \mathrm{O}_{3}\left[\mathrm{M}^{+}\right]: 280.0615$, found: 280.0612 .

\section{(4-chloro-2,6-dimethoxypyrimidin-5-yl)(2-methoxyphenyl)methanol (8b).}

A dry and argon flushed $10 \mathrm{~mL}$ flask, equipped with a magnetic stirrer and a septum, was charged with a solution of 5-bromo-4-chloro-2,6-dimethoxypyrimidine (4) (254 mg, $1 \mathrm{mmol}$ ) in dry THF $(1 \mathrm{~mL}) . i$-PrMgCl$\cdot \mathrm{LiCl}(1.0 \mathrm{M} / \mathrm{THF}, 1.05 \mathrm{mmol}, 1.05$ equiv) was added slowly at room temperature and the resulting mixture was stirred for $15 \mathrm{~min}$ to complete the brominemagnesium exchange (checked by GC-MS analysis of reaction aliquots). 2methoxybenzaldehyde (150 mg, $1.1 \mathrm{mmol}, 1.1$ equiv) was added in one portion. The mixture 
was stirred for $10 \mathrm{~h}$ and was quenched with saturated aqueous $\mathrm{NH}_{4} \mathrm{Cl}$ solution. The aqueous phase was extracted with ether $(3 \times 5 \mathrm{~mL})$. The organic fractions were dried $\left(\mathrm{Na}_{2} \mathrm{SO}_{4}\right)$ and concentrated in vacuo. Purification by flash chromatography ( $n$-pentane/diethyl ether $=3: 2$ ) yielded $258 \mathrm{mg}$ (83\% yield) of $\mathbf{8 b}$ as a white solid (mp.: 106.0-107.3 $\left.{ }^{\circ} \mathrm{C}\right)$.

${ }^{1} \mathrm{H}-\mathrm{NMR}\left(\mathrm{CDCl}_{3}, 400 \mathrm{MHz}\right): \delta=7.34(\mathrm{~d}, J=7.6 \mathrm{~Hz}, 1 \mathrm{H}), 7.26(\mathrm{t}, J=8.8 \mathrm{~Hz}, 1 \mathrm{H}), 6.94(\mathrm{t}, J$ $=7.5 \mathrm{~Hz}, 1 \mathrm{H}), 6.84(\mathrm{~d}, J=8.2 \mathrm{~Hz}, 1 \mathrm{H}), 6.38(\mathrm{~d}, J=8.4 \mathrm{~Hz}, 1 \mathrm{H}), 3.98(\mathrm{~s}, 3 \mathrm{H}), 3.95(\mathrm{~s}, 3 \mathrm{H})$, $3.79(\mathrm{~s}, 3 \mathrm{H}), 3.33(\mathrm{~d}, J=8.8 \mathrm{~Hz}, 1 \mathrm{H})$.

${ }^{13} \mathrm{C}-\mathrm{NMR}\left(\mathrm{CDCl}_{3}, 100 \mathrm{MHz}\right): 170.13,163.11,156.76,129.36,128.90,127.07,120.22$, $113.53,110.60,66.75,55.55,55.51,55.00$.

MS (EI, $70 \mathrm{ev),} \mathrm{m/z} \mathrm{( \% ):} 312.2$ (22), 311.2 (15), 310.2 (58), 294.1 (38), 293.1 (23), 292.1 (100), 257.12 (57), 242.1 (12), 205.1 (13), 203.1 (51), 201.1 (53), 175.1 (29), 135.1 (16), 109.1 (17), 108.1 (19), 77.1 (10).

IR (KBr): 3472 (s), 3003 (m), 2963 (w), 1553 (s), 1455 (m), 1375 (s), 1241 (m), 1206 (m), $1021(\mathrm{~s})$.

HRMS (EI): calcd. for $\mathrm{C}_{14} \mathrm{H}_{15} \mathrm{ClN}_{2} \mathrm{O}_{4}\left[\mathrm{M}^{+}\right]$: 310.0720, found: 310.0733 .

\section{4-chloro-2,6-dimethoxypyrimidin-5-yl)(phenyl)methanone (8c).}

A dry and argon flushed $10 \mathrm{~mL}$ flask, equipped with a magnetic stirrer and a septum, was charged with a solution of 5-bromo-4-chloro-2,6-dimethoxypyrimidine (4) (254 mg, $1 \mathrm{mmol}$ ) in dry THF $(1 \mathrm{~mL}) . i$-PrMgCl$\cdot \mathrm{LiCl}(1.0 \mathrm{M} / \mathrm{THF}, 1.05 \mathrm{mmol}, 1.05$ equiv) was added slowly at room temperature and the resulting mixture was stirred for $15 \mathrm{~min}$ to complete the brominemagnesium exchange (checked by GC-MS analysis of reaction aliquots) and cooled down to $-20{ }^{\circ} \mathrm{C}$. Benzoyl chloride (154 mg, $1.1 \mathrm{mmol}, 1.1$ equiv) was added dropwise. The mixture was warmed up at room temperature for $3 \mathrm{~h}$ and was quenched with saturated aqueous $\mathrm{NH}_{4} \mathrm{Cl}$ solution. The aqueous phase was extracted with ether $(3 \times 5 \mathrm{~mL})$. The organic fractions were dried $\left(\mathrm{Na}_{2} \mathrm{SO}_{4}\right)$ and concentrated in vacuo. Purification by flash chromatography ( $n$ pentane/diethyl ether= 3:2) yielded $231 \mathrm{mg}$ (86\% yield) of 8c as a white solid (mp.: 116.0$\left.117.3^{\circ} \mathrm{C}\right)$.

${ }^{1} \mathrm{H}-\mathrm{NMR}\left(\mathrm{CDCl}_{3}, 300 \mathrm{MHz}\right): \delta=7.83(\mathrm{~d}, J=8.5 \mathrm{~Hz}, 2 \mathrm{H}), 7.62(\mathrm{t}, J=7.3 \mathrm{~Hz}, 1 \mathrm{H}), 7.48(\mathrm{t}, J$ $=7.8 \mathrm{~Hz}, 2 \mathrm{H}), 4.07(\mathrm{~s}, 3 \mathrm{H}), 3.94(\mathrm{~s}, 3 \mathrm{H})$.

${ }^{13} \mathrm{C}-\mathrm{NMR}\left(\mathrm{CDCl}_{3}, 75 \mathrm{MHz}\right): 190.69,169.47,164.27,158.11,136.17,134.19,129.45,128.85$, $112.81,55.68,55.11$.

MS (EI, $70 \mathrm{ev}), \mathrm{m} / z$ (\%): 280.2 (17), 278.2 (47), 203.1 (34), 201.1 (100), 105.1 (30), 77.1 (17), $76.1(11)$. 
IR (KBr): 3436 (m), 3000 (w), 2952 (w), 1676 (s), 1589 (s), 1485 (s), 1485 (m), 1389 (s), 1200 (s), 1027 (m), 921 (m).

HRMS (EI): calcd. for $\mathrm{C}_{13} \mathrm{H}_{11} \mathrm{ClN}_{2} \mathrm{O}_{3}\left[\mathrm{M}^{+}\right]: 278.0458$, found: 278.0441 .

\section{(4-chloro-2,6-dimethoxypyrimidin-5-yl)(morpholino)methanone( 8d).}

A dry and argon flushed $10 \mathrm{~mL}$ flask, equipped with a magnetic stirrer and a septum, was charged with a solution of 5-bromo-4-chloro-2,6-dimethoxypyrimidine (4) (254 mg, $1 \mathrm{mmol}$ ) in dry THF $(1 \mathrm{~mL}) . i$-PrMgCl$\cdot \mathrm{LiCl}(1.0 \mathrm{M} / \mathrm{THF}, 1.05 \mathrm{mmol}, 1.05$ equiv) was added slowly at room temperature and the resulting mixture was stirred for $15 \mathrm{~min}$ to complete the brominemagnesium exchange (checked by GC-MS analysis of reaction aliquots) and cooled down to $-20{ }^{\circ} \mathrm{C}$. 4-morpholinecarbonyl chloride (165 mg, $1.1 \mathrm{mmol}, 1.1$ equiv) was added dropwise. The mixture was warmed up to room temperature for $15 \mathrm{~h}$ and was quenched with saturated aqueous $\mathrm{NH}_{4} \mathrm{Cl}$ solution. The aqueous phase was extracted with ether $(3 \times 5 \mathrm{~mL})$. The organic fractions were dried $\left(\mathrm{Na}_{2} \mathrm{SO}_{4}\right)$ and concentrated in vacuo. Purification by flash chromatography ( $n$-pentane/diethyl ether $=2: 3)$ yielded $244 \mathrm{mg}(85 \%$ yield $)$ of $\mathbf{8 d}$ as a white solid (mp.: 109.9-111.3 $\left.{ }^{\circ} \mathrm{C}\right)$.

${ }^{1} \mathrm{H}-\mathrm{NMR}$ (DMSO, $400 \mathrm{MHz}$ ): $\delta=4.01$ (2 singulets, 6H), 3.86-3.61 (3 multiplets, $6 \mathrm{H}$ ), 3.28 $(\mathrm{m}, 2 \mathrm{H})$.

${ }^{13}$ C-NMR (DMSO, 100 MHz): 168.55, 164.12, 162.02, 157.89, 109.39, 66.75, 66.56, 55.63, $55.21,46.92,42.20$.

MS (EI, $70 \mathrm{ev),} \mathrm{m/z} \mathrm{( \% ):} 287.0$ (9), 252.1 (11), 203.0 (28), 201.0 (100), 75.9 (11).

IR (KBr): 2960 (w), 2862 (w), 1634 (m), 1580 (s), 1534 (s), 1494 (m), 1370 (s), 1357 (m), 1275 (m), 1235 (s), 1111 (s), 1009 (s).

HRMS (EI): calcd. for $\mathrm{C}_{11} \mathrm{H}_{14} \mathrm{ClN}_{3} \mathrm{O}_{4}\left[\mathrm{M}^{+}\right]$: 287.0673, found: 287.0664 .

\section{4-chloro-2,6-dimethoxypyrimidine-5-carbonitrile (8e).}

A dry and argon flushed $10 \mathrm{~mL}$ flask, equipped with a magnetic stirrer and a septum, was charged with a solution of 5-bromo-4-chloro-2,6-dimethoxypyrimidine (4) (254 mg, $1 \mathrm{mmol}$ ) in dry THF (1 mL). i-PrMgCl-LiCl (1.0 M/THF, $1.05 \mathrm{mmol}, 1.05$ equiv) was added slowly at room temperature and the resulting mixture was stirred for $15 \mathrm{~min}$ to complete the brominemagnesium exchange (checked by GC-MS analysis of reaction aliquots). p-toluenesulfonyl cyanide (200 mg, $1.1 \mathrm{mmol}, 1.1$ equiv) was added in one portion. The mixture was stirred overnight (12 h) at rt and was quenched with saturated aqueous $\mathrm{NH}_{4} \mathrm{Cl}$ solution. The aqueous phase was extracted with ethyl acetate $(2 \times 5 \mathrm{~mL})$ and dichloromethane $(1 \times 5 \mathrm{~mL})$. The 
organic fractions were dried $\left(\mathrm{Na}_{2} \mathrm{SO}_{4}\right)$ and concentrated in vacuo. Purification by flash chromatography $(n$-pentane/diethyl ether $=1: 4)$ yielded $178 \mathrm{mg}(89 \%$ yield $)$ of $\mathbf{8 e}$ as a white solid (mp.: $\left.123.3-124.8^{\circ} \mathrm{C}\right)$.

${ }^{1} \mathrm{H}-\mathrm{NMR}\left(\mathrm{CDCl}_{3}, 400 \mathrm{MHz}\right): \delta=4.11(\mathrm{~s}, 3 \mathrm{H}), 4.07$ (s, 3H).

${ }^{13} \mathrm{C}-\mathrm{NMR}\left(\mathrm{CDCl}_{3}, 100 \mathrm{MHz}\right): 169.24,163.98,163.41,158.85,108.67,62.14,55.67,55.23$, 14.01 .

MS (EI, $70 \mathrm{ev),} \mathrm{m/z} \mathrm{( \% ):} 201.1$ (25), 199.1 (71), 198.1 (32), 171.1 (33), 169.1 (100), 154.1 (32), 106.1 (19), 70.1 (11).

IR (KBr): 3021 (w), 2947 (w), 2233 (m), 1586 (m), 1529 (s), 1474 (m), 1378 (m), 1218 (m), $1071(\mathrm{~m}), 1018$ (s).

HRMS (EI): calcd. for $\mathrm{C}_{7} \mathrm{H}_{6} \mathrm{ClN}_{3} \mathrm{O}_{2}\left[\mathrm{M}^{+}\right]$: 199.0149, found: 199.0131.

\section{Ethyl 4-chloro-2,6-dimethoxypyrimidine-5-carboxylate (8f).}

A dry and argon flushed $10 \mathrm{~mL}$ flask, equipped with a magnetic stirrer and a septum, was charged with a solution of 5-bromo-4-chloro-2,6-dimethoxypyrimidine (4) (381 mg, 1.5 $\mathrm{mmol})$ in dry THF (2 mL), $i$-PrMgCl-LiCl (1.0 M/THF, $1.58 \mathrm{mmol}, 1.05$ equiv) was added slowly at room temperature and the resulting mixture was stirred for 15 min to complete the bromine-magnesium exchange (checked by GC-MS analysis of reaction aliquots). The mixture was cooled down to $-20^{\circ} \mathrm{C}$ and ethyl cyanoformate (164 mg, 1.1 equiv) was added dropwise. The mixture was stirred at $-20{ }^{\circ} \mathrm{C}$ for $10 \mathrm{~min}$, warmed up to room temperature for 8 $h$ and was quenched with saturated aqueous $\mathrm{NH}_{4} \mathrm{Cl}$ solution. The aqueous phase was extracted with ethyl acetate $(3 \times 10 \mathrm{~mL})$. The organic fractions were dried $\left(\mathrm{Na}_{2} \mathrm{SO}_{4}\right)$ and concentrated in vacuo. Purification by flash chromatography ( $n$-pentane/diethyl ether $=4: 1$ ) yielded 322 $\mathrm{mg}(87 \%$ yield) of $\mathbf{8 f}$ as a yellow oil.

${ }^{1} \mathrm{H}-\mathrm{NMR}\left(\mathrm{CDCl}_{3}, 400 \mathrm{MHz}\right): \delta=4.40(\mathrm{q}, J=7.1 \mathrm{~Hz}, 2 \mathrm{H}), 4.02(\mathrm{~s}, 6 \mathrm{H}), 1.38(\mathrm{t}, J=7.1 \mathrm{~Hz}$, $3 \mathrm{H})$.

${ }^{13} \mathrm{C}-\mathrm{NMR}\left(\mathrm{CDCl}_{3}, 100 \mathrm{MHz}\right): 172.22,165.12,164.89,111.75,89.77,56.39,55.96$.

MS (EI, $70 \mathrm{ev),} \mathrm{m/z} \mathrm{( \% ):} 246.2$ (15), 218.1 (13), 203.1 (30), 201.1 (100), 174.1 (13), 76.1 (14).

IR (film): 2957 (w), 1731 (s), 1585 (s), 1540 (s), 1488 (m), 1376 (m), 1218 (m), 1071 (m), $1018(\mathrm{~s})$.

HRMS (EI): calcd. for $\mathrm{C}_{7} \mathrm{H}_{6} \mathrm{ClN}_{3} \mathrm{O}_{2}\left[\mathrm{M}^{+}\right]$: 246.0407, found: 246.0388 . 


\section{5-benzyl-4-chloro-2,6-dimethoxypyrimidine (8g).}

A dry and argon flushed $10 \mathrm{~mL}$ flask, equipped with a magnetic stirrer and a septum, was charged with a solution of 5-bromo-4-chloro-2,6-dimethoxypyrimidine (4) (381 mg, 1.5 $\mathrm{mmol})$ in dry THF (2 mL). $i$-PrMgCl.LiCl (1.0 M/THF, $1.58 \mathrm{mmol}, 1.05$ equiv) was added slowly at room temperature and the resulting mixture was stirred for $15 \mathrm{~min}$ to complete the bromine-magnesium exchange (checked by GC-MS analysis of reaction aliquots). Benzylbromide (360 mg, $2.1 \mathrm{mmol}, 1.4$ equiv) was added dropwise. The mixture was stirred at $\mathrm{rt}$ for $4 \mathrm{~h}$ and was quenched with saturated aqueous $\mathrm{NH}_{4} \mathrm{Cl}$ solution. The aqueous phase was extracted with ethyl acetate $(3 \times 5 \mathrm{~mL})$. The organic fractions were dried $\left(\mathrm{Na}_{2} \mathrm{SO}_{4}\right)$ and concentrated in vacuo. Purification by flash chromatography (n-pentane/diethyl ether $=1: 4$ ) yielded $300 \mathrm{mg}$ (75\% yield) of $\mathbf{8 g}$ as a colorless oil.

${ }^{1} \mathrm{H}-\mathrm{NMR}\left(\mathrm{CDCl}_{3}, 400 \mathrm{MHz}\right): \delta=7.14(\mathrm{~m}, 5 \mathrm{H}), 3.90-3.91(\mathrm{~m}, 8 \mathrm{H})$.

${ }^{13} \mathrm{C}-\mathrm{NMR}\left(\mathrm{CDCl}_{3}, 100 \mathrm{MHz}\right)$ : 170.26, 162.65, 160.23, 138.57, 128.30, 126.29, 112.04, 55.13, $54.82,31.16$.

MS (EI, $70 \mathrm{ev}), \mathrm{m} / z$ (\%): 266.2 (31), 264.2 (100), 249.1 (33), 234.2 (19), 173.1 (17), 156.1 (10), $91.1(18), 77.1(8)$.

IR (film): 3028 (w), 2950 (w), 1586 (m), 1541 (s), 1453 (m), 1369 (s), 1213 (m), 1078 (m), 1026 (s).

HRMS (EI): calcd. for $\mathrm{C}_{13} \mathrm{H}_{13} \mathrm{ClN}_{2} \mathrm{O}_{2}\left[\mathrm{M}^{+}\right]$: 264.0666, found: 264.0639 .

\section{4-bromo-2,6-dimethoxy-5-(trimethylsilyl)pyrimidine (9a).}

A dry and argon flushed $10 \mathrm{~mL}$ flask, equipped with a magnetic stirrer and a septum, was charged with a solution of 4,5-dibromo-2,6-dimethoxypyrimidine (5) (596 mg, 2 mmol) in dry THF (3 mL). $i$-PrMgCl-LiCl (1.0 M/THF, $2.1 \mathrm{mmol}, 1.05$ equiv) was added very slowly at room temperature and the resulting mixture was stirred for 15 min to complete the brominemagnesium exchange (checked by GC-MS analysis of reaction aliquots). Trimethylsilyl chloride (240 mg, $2.2 \mathrm{mmol}, 1.1$ equiv) was added dropwise. The mixture was stirred at $\mathrm{rt}$ for $24 \mathrm{~h}$ and was quenched with saturated aqueous $\mathrm{NH}_{4} \mathrm{Cl}$ solution. The aqueous phase was extracted with ethyl acetate $(2 \times 10 \mathrm{~mL})$. The organic fractions were dried $\left(\mathrm{Na}_{2} \mathrm{SO}_{4}\right)$ and concentrated in vacuo. Purification by flash chromatography (n-pentane/diethyl ether $=4: 1$ ) yielded $529 \mathrm{mg}$ (91\% yield) of 9a as a white solid (mp.: 71.1-72.2 ${ }^{\circ} \mathrm{C}$ ).

${ }^{1} \mathrm{H}-\mathrm{NMR}\left(\mathrm{CDCl}_{3}, 400 \mathrm{MHz}\right): \delta=3.98(\mathrm{~s}, 3 \mathrm{H}), 3.92$ (s, 3H), 0.37 (s, 9H).

${ }^{13} \mathrm{C}-\mathrm{NMR}\left(\mathrm{CDCl}_{3}, 100 \mathrm{MHz}\right): 175.51,164.45,159.89,111.76,55.05,54.13,1.24$. 
MS (EI, $70 \mathrm{ev),} \mathrm{m/z} \mathrm{( \% ):} 292.1$ (10), 290.1 (10), 277.1 (100), 275.1 (99), 247.1 (81), 220.1 (30), 194.1 (13), $137.0(33), 72.1$ (38).

IR (KBr): 2955 (w), 2899 (w), 1556 (m), 1524 (s), 1450 (m), 1344 (s), 1278 (m), 1114 (m), $1015(\mathrm{~m}), 839$ (s).

HRMS (EI): calcd. for $\mathrm{C}_{9} \mathrm{H}_{15} \mathrm{BrN}_{2} \mathrm{O}_{2} \mathrm{Si}\left[\mathrm{M}^{+}\right]$: 290.0086, found: 290.0062 .

\section{5-allyl-4-bromo-2,6-dimethoxypyrimidine (9b).}

A dry and argon flushed $10 \mathrm{~mL}$ flask, equipped with a magnetic stirrer and a septum, was charged with a solution of 4,5-dibromo-2,6-dimethoxypyrimidine (5) (596 mg, $2 \mathrm{mmol}$ ) in dry THF (3 mL). $i$-PrMgCl-LiCl (1.0 M/THF, $2.1 \mathrm{mmol}, 1.05$ equiv) was added very slowly (within $5 \mathrm{~min}$ ) at room temperature and the resulting mixture was stirred for $15 \mathrm{~min}$ to complete the bromine-magnesium exchange (checked by GC-MS analysis of reaction aliquots). Allyl bromide ( $266 \mathrm{mg}, 2.2 \mathrm{mmol}, 1.1$ equiv) was added dropwise. The mixture was stirred at $\mathrm{rt}$ for $2 \mathrm{~h}$ and was quenched with saturated aqueous $\mathrm{NH}_{4} \mathrm{Cl}$ solution. The aqueous phase was extracted with ethyl acetate $(2 \times 5 \mathrm{~mL})$. The organic fractions were dried $\left(\mathrm{Na}_{2} \mathrm{SO}_{4}\right)$ and concentrated in vасио. Purification by flash chromatography (n-pentane/diethyl ether = 9:1) yielded $528 \mathrm{mg}$ (91\% yield) of $\mathbf{9 b}$ as a colorless oil.

${ }^{1} \mathrm{H}-\mathrm{NMR}\left(\mathrm{CDCl}_{3}, 400 \mathrm{MHz}\right): \delta=5.82(\mathrm{~m}, 1 \mathrm{H}), 5.02\left(2 \mathrm{dd},{ }^{3} J_{\text {trans }}=13.2 \mathrm{~Hz}, J_{\text {gem }}\right.$ and ${ }^{3} J_{\text {cis }}=$ $1.7 \mathrm{~Hz}, 2 \mathrm{H}), 3.98$ (s, 3H), 3.97 (s, 3H), $3.35\left(\mathrm{dt},{ }^{3} \mathrm{~J}=6.1 \mathrm{~Hz},{ }^{4} \mathrm{~J}=1.5 \mathrm{~Hz}\right)$.

${ }^{13} \mathrm{C}-\mathrm{NMR}\left(\mathrm{CDCl}_{3}, 100 \mathrm{MHz}\right): 170.08,162.61,159.98,133.39,115.93,110.71,55.14,54.82$, 29.57 .

MS (EI, $70 \mathrm{ev),} \mathrm{m/z} \mathrm{( \% ):} 260.0$ (98), 258.0 (100), 245.0 (23), 243.0 (22), 231.0 (41), 179.0 (25), $163.0(14)$.

IR (film): 3081 (w), 2957 (w), 1639 (w), 1586 (m), 1542 (s), 1459 (m), 1370 (s), 1223 (m), $1079(\mathrm{~m}), 1026(\mathrm{~s})$.

HRMS (EI): calcd. for $\mathrm{C}_{9} \mathrm{H}_{11} \mathrm{BrN}_{2} \mathrm{O}_{2}\left[\mathrm{M}^{+}\right]$: 258.0004, found: 258.0023 .

\section{Ethyl 4-bromo-2,6-dimethoxypyrimidine-5-carboxylate (9c).}

A dry and argon flushed $10 \mathrm{~mL}$ flask, equipped with a magnetic stirrer and a septum, was charged with a solution of 5-bromo-4-chloro-2,6-dimethoxypyrimidine (5) (298 mg, 1.0 $\mathrm{mmol})$ in dry THF (2 mL), $i$-PrMgCl$\cdot \mathrm{LiCl}(1.0 \mathrm{M} / \mathrm{THF}, 1.05 \mathrm{mmol}, 1.05$ equiv) was added very slowly (within $5 \mathrm{~min}$ ) at room temperature and the resulting mixture was stirred for 15 min to complete the bromine-magnesium exchange (checked by GC-MS analysis of reaction aliquots) and cooled down to $-20{ }^{\circ} \mathrm{C}$. Ethyl cyanoformate (164 mg, 1.1 equiv) was added 
dropwise. The mixture was stirred at $-20{ }^{\circ} \mathrm{C}$ for $1 \mathrm{~h}$, warmed up to room temperature for $12 \mathrm{~h}$ and was quenched with saturated aqueous $\mathrm{NH}_{4} \mathrm{Cl}$ solution. The aqueous phase was extracted with ethyl acetate $(3 \times 5 \mathrm{~mL})$. The organic fractions were dried $\left(\mathrm{Na}_{2} \mathrm{SO}_{4}\right)$ and concentrated in vасио. Purification by flash chromatography ( $n$-pentane/diethyl ether $=4: 1$ ) yielded $236 \mathrm{mg}$ (81\% yield) of $9 \mathbf{c}$ as a white solid (mp.: $42.5-43.8^{\circ} \mathrm{C}$ ).

${ }^{1} \mathrm{H}-\mathrm{NMR}\left(\mathrm{CDCl}_{3}, 400 \mathrm{MHz}\right): \delta=4.38(\mathrm{q}, J=7.1 \mathrm{~Hz}, 2 \mathrm{H}), 4.01(\mathrm{~s}, 6 \mathrm{H}), 1.37(\mathrm{t}, J=7.1 \mathrm{~Hz}$, $3 \mathrm{H})$.

${ }^{13} \mathrm{C}-\mathrm{NMR}\left(\mathrm{CDCl}_{3}, 100 \mathrm{MHz}\right): 166.52,164.00,163.61,149.96,112.0,62.21,55.69,55.15$, 13.98 .

MS (EI, $70 \mathrm{ev),} \mathrm{m/z} \mathrm{( \% ):} 292.1$ (18), 290.1 (19), 247.1 (96), 245.1 (100), 218.1 (18), 151.1 (8), 122.0 (19), 70.1 (11).

IR (KBr): 2983 (w), 2960 (w), 1725 (s), 1570 (s), 1529 (s), 1491 (m), 1358 (m), 1265 (s), 1229 (s), 1048 (m), 1013 (s).

HRMS (EI): calcd. for $\mathrm{C}_{9} \mathrm{H}_{11} \mathrm{BrN}_{2} \mathrm{O}_{4}\left[\mathrm{M}^{+}\right]$: 289.9902, found: 289.9902 .

\section{(4-bromo-2,6-dimethoxypyrimidin-5-yl)(phenyl)methanol (9d).}

A dry and argon flushed $10 \mathrm{~mL}$ flask, equipped with a magnetic stirrer and a septum, was charged with a solution of 4,5-dibromo-2,6-dimethoxypyrimidine (5) (298 mg, $1.0 \mathrm{mmol}$ ) in dry THF (2 mL). $i$-PrMgCl-LiCl (1.0 M/THF, $1.05 \mathrm{mmol}, 1.05$ equiv) was added very slowly (within 5min) at room temperature and the resulting mixture was stirred for $15 \mathrm{~min}$ to complete the bromine-magnesium exchange (checked by GC-MS analysis of reaction aliquots). Benzaldehyde (117 mg, $1.1 \mathrm{mmol}, 1.1$ equiv) was added dropwise. The mixture was stirred at $\mathrm{rt}$ for $4 \mathrm{~h}$ and was quenched with saturated aqueous $\mathrm{NH}_{4} \mathrm{Cl}$ solution. The aqueous phase was extracted with ethyl acetate $(3 \times 5 \mathrm{~mL})$. The organic fractions were dried $\left(\mathrm{Na}_{2} \mathrm{SO}_{4}\right)$ and concentrated in vacuo. Purification by flash chromatography (n-pentane/diethyl ether = 3:2) yielded $308 \mathrm{mg}\left(95 \%\right.$ yield) of $9 \mathbf{d}$ as a white solid (mp.: 108.9-111.2 ${ }^{\circ} \mathrm{C}$ ).

${ }^{1} \mathrm{H}-\mathrm{NMR}\left(\mathrm{CDCl}_{3}, 400 \mathrm{MHz}\right): \delta=7.3(\mathrm{~m}, 5 \mathrm{H}), 6.19(\mathrm{~d}, J=11.1 \mathrm{~Hz}, 1 \mathrm{H}), 4.01(\mathrm{~s}, 3 \mathrm{H}), 3.93$ (s, $3 \mathrm{H}), 3.41(\mathrm{~d}, J=11.1 \mathrm{~Hz}, 1 \mathrm{H})$.

${ }^{13} \mathrm{C}-\mathrm{NMR}\left(\mathrm{CDCl}_{3}, 100 \mathrm{MHz}\right): 168.95,162.95,152.53,141.65,128.32,127.47,125.29$, $116.65,55.42,54.96$.

MS (EI, $70 \mathrm{ev),} \mathrm{m/z} \mathrm{( \% ):} 326.1$ (41), 324.1 (33), 308.1 (18), 306.1 (18), 249.1 (67), 247.1 (100), 227.1 (32), 105.1 (17), 77.1 (22).

IR (KBr): 3337 (s), 2953 (w), 2923 (w), 1572 (w), 1543 (s), 1488 (m), 1448 (m), 1368 (s), $1212(\mathrm{~m}), 1016(\mathrm{~s})$. 
HRMS (EI): calcd. for $\mathrm{C}_{13} \mathrm{H}_{13} \mathrm{BrN}_{2} \mathrm{O}_{3}\left[\mathrm{M}^{+}\right]$: 324.0110, found: 324.0092 .

\section{(4-bromo-2,6-dimethoxypyrimidin-5-yl)(morpholino)methanone (9e).}

A dry and argon flushed $10 \mathrm{~mL}$ flask, equipped with a magnetic stirrer and a septum, was charged with a solution of 5-bromo-4-chloro-2,6-dimethoxypyrimidine (5) (298 mg, $1 \mathrm{mmol}$ ) in dry THF (2 mL). $i$-PrMgCl-LiCl (1.0 M/THF, $1.05 \mathrm{mmol}, 1.05$ equiv) was added slowly at room temperature and the resulting mixture was stirred for $15 \mathrm{~min}$ to complete the brominemagnesium exchange (checked by GC-MS analysis of reaction aliquots. 4morpholinecarbonyl chloride (165 mg, $1.1 \mathrm{mmol}, 1.1$ equiv) was added dropwise. The mixture was stirred at room temperature for $12 \mathrm{~h}$ and was quenched with saturated aqueous $\mathrm{NH}_{4} \mathrm{Cl}$ solution. The aqueous phase was extracted with ethyl acetate $(3 \times 5 \mathrm{~mL})$. The organic fractions were dried $\left(\mathrm{Na}_{2} \mathrm{SO}_{4}\right)$ and concentrated in vacuo. Purification by flash chromatography ( $n$-pentane/diethyl ether $=2: 3)$ yielded $210 \mathrm{mg}(70 \%$ yield $)$ of $9 \mathrm{e}$ as a white solid (mp.: 112.0-113.6 $\left.{ }^{\circ} \mathrm{C}\right)$.

${ }^{1} \mathrm{H}-\mathrm{NMR}$ (DMSO, $\left.400 \mathrm{MHz}\right): \delta=3.95$ (s, 3H), 3.94 (s, 3H), 3.57 (m, 6H), 3.27 (m, 2H ).

${ }^{13} \mathrm{C}-\mathrm{NMR}$ (DMSO, $100 \mathrm{MHz}$ ): 167.20, 163.11, 161.58, 148.66, 112.54, 66.25, 55.27, 54.95, 46.27, 41.63 .

MS (EI, $70 \mathrm{ev}), \mathrm{m} / \mathrm{z}(\%): 333.0$ (8), 331.0 (9), 252.1 (30), 247.1 (98), 245.1 (100), 167.2 (12), $122.1(11), 120.1$ (11), $70.1(6)$.

IR (KBr): 2960 (w), 2862 (w), 1634 (m), 1580 (s), 1534 (s), 1494 (m), 1370 (s), 1357 (m), 1275 (m), 1235 (s), 1111 (s), 1009 (s).

HRMS (EI): calcd. for $\mathrm{C}_{11} \mathrm{H}_{14} \mathrm{BrN}_{3} \mathrm{O}_{4}\left[\mathrm{M}^{+}\right]$: 331.0168, found: 331.0177 .

\section{Synthesis of annelated heterocycles 10 and 11:}

\section{4,6-dimethoxy-3-phenylisoxazolo[5,4-d]pyrimidine (10).}

To a stirring solution of 4-chloro-2,6-dimethoxypyrimidin-5-yl)(phenyl)methanone (8c) (1.39 $\mathrm{mg}, 5 \mathrm{mmol})$ in $\mathrm{EtOH} 50 \%(100 \mathrm{~mL})$ at $\mathrm{rt}$ was added hydroxylamine hydrochloride $(1.04 \mathrm{~g}$, $15 \mathrm{mmol})$ and sodium acetate $(1.25 \mathrm{~g}, 15 \mathrm{mmol})$. The mixture was refluxing during $4 \mathrm{~h}$. The product was collected by filtration and washed two times with $\mathrm{H}_{2} \mathrm{O}(10 \mathrm{~mL})$. Purification by flash chromatography ( $n$-pentane/diethyl ether $=3: 2)$ yielded $1.88 \mathrm{~g}$ (83\% yield) of $\mathbf{1 0}$ as a white solid (mp.: 210,1-211.9 ${ }^{\circ} \mathrm{C}$ ) .

${ }^{1} \mathrm{H}-\mathrm{NMR}$ (DMSO, $\left.400 \mathrm{MHz}\right): \delta=8.20(\mathrm{~m}, 2 \mathrm{H}), 7.60(\mathrm{~m}, 3 \mathrm{H}), 4.14(\mathrm{~s}, 3 \mathrm{H}), 4.00(\mathrm{~s}, 3 \mathrm{H})$. 
${ }^{13} \mathrm{C}-\mathrm{NMR}$ (DMSO, $100 \mathrm{MHz}$ ): 166.79, 167.63, 166.76, 132.25, 132.22, 129.16, 128.30, $125.98,96.27,55.37,55.06$.

MS (EI, $70 \mathrm{ev),} \mathrm{m/z} \mathrm{( \% ):} 257.1$ (100), 227.1 (57), 212.1 (36), 105.0 (65), 91.0 (8), 77.0 (41), 70.0 (12), $51.0(11)$.

IR (KBr): 3017 (w), 2951 (w), 1615 (s), 1539 (s), 1441 (s), 1385 (m), 1318 (s), 1138 (m). HRMS (EI): calcd. for $\mathrm{C}_{13} \mathrm{H}_{11} \mathrm{~N}_{3} \mathrm{O}_{3}\left[\mathrm{M}^{+}\right]$: 257.0800, found: 257.0807 .

\section{methyl 2,4-dimethoxy-5-phenylthieno[2,3-d]pyrimidine-6-carboxylate (11).}

Triethylamine (0.4 g, $4 \mathrm{mmol})$ was added dropwise to a mixture of 4-chloro-2,6dimethoxypyrimidin-5-yl)(phenyl)methanone (8c) $(0.55 \mathrm{~g}, 2 \mathrm{mmol})$ and methyl mercaptoacetate $(0,31 \mathrm{~g}, 2.6 \mathrm{mmol})$ in $\mathrm{EtOH}(10 \mathrm{~mL})$. The reaction mixture was heated at reflux until the starting ketone 8c disappeared according to gas chromatography (12 h). After cooling to room temperature, the precipitate was filtered off and recrystallized in $\mathrm{EtOH} 95 \%$ to give a white powder 11 (mp: $193.3-194.3^{\circ} \mathrm{C}$ ) in $69 \%$ yield.

${ }^{1} \mathrm{H}-\mathrm{NMR}\left(\mathrm{CDCl}_{3}, 300 \mathrm{MHz}\right): \delta=7.40(\mathrm{~m}, 3 \mathrm{H}), 7.38(\mathrm{~m}, 2 \mathrm{H}), 4.08(\mathrm{~s}, 3 \mathrm{H}), 3.79(\mathrm{~s}, 3 \mathrm{H}), 3.72$ $(\mathrm{s}, 3 \mathrm{H})$.

${ }^{13} \mathrm{C}-\mathrm{NMR}\left(\mathrm{CDCl}_{3}, 75 \mathrm{MHz}\right)$ : 170.19, 167.23, 163.85, 162.25, 142.15, 134.89, 129.05, 127.84, 127.20, 122.47, 114.90, 55.33, 54.10, 52.16.

MS (EI, $70 \mathrm{ev),} \mathrm{m/z} \mathrm{( \% ):} 331.1$ (16), 330.1 (100), 329.1 (15), 300.1 (11), 299.1 (26), 284.0 (11), 251.9 (7), 227.0 (8), 199.0 (7), 171.0 (6), $127.0(8)$.

IR (KBr): 3012 (w), 2951 (w), 1716 (s), 1577 (m), 1541 (s), 1473 (m), 1249 (s), 1168 (m), $1027(\mathrm{~m})$.

HRMS (EI): calcd. for $\mathrm{C}_{16} \mathrm{H}_{14} \mathrm{~N}_{2} \mathrm{O}_{4} \mathrm{~S}\left[\mathrm{M}^{+}\right]$: 330.0674 , found: 330.0660 .

\section{Synthesis of oxypurinol (2):}

\section{4-chloro-2,6-dimethoxypyrimidine-5-carbaldehyde (12).}

A dry and argon flushed $10 \mathrm{~mL}$ flask, equipped with a magnetic stirrer and a septum, was charged with a solution of 5-bromo-4-chloro-2,6-dimethoxypyrimidine (4) (1.27 g, $5 \mathrm{mmol})$ in dry THF ( $5 \mathrm{~mL}) . i$-PrMgCl$\cdot \mathrm{LiCl}(1.0 \mathrm{M} / \mathrm{THF}, 5.25 \mathrm{mmol}, 1.05$ equiv) was added slowly at room temperature and the resulting mixture was stirred for 15 min to complete the brominemagnesium exchange (checked by GC-MS analysis of reaction aliquots). The mixture was cooled down to $-35^{\circ} \mathrm{C}$ and 4 -formylmorpholine ( $0.635 \mathrm{~g}, 1.1$ equiv) was added dropwise. The mixture was stirred at $-35^{\circ} \mathrm{C}$ for $4 \mathrm{~h}$ and was quenched with a mixture of a solution of acetic 
acid in water $(\approx 1 \mathrm{M} ; 3 \mathrm{~mL})$ and saturated aqueous $\mathrm{NH}_{4} \mathrm{Cl}$ solution $(15 \mathrm{~mL})$. The aqueous phase was extracted with ether $(3 \times 20 \mathrm{~mL})$. The organic fractions were dried $\left(\mathrm{Na}_{2} \mathrm{SO}_{4}\right)$ and concentrated in vacuo. Purification by flash chromatography ( $n$-pentane/diethyl ether $=3: 2$ ) yielded $840 \mathrm{mg}$ ( $83 \%$ yield) of $\mathbf{1 2}$ as a white solid (mp.: 97.8-99. $\left.1^{\circ} \mathrm{C}\right)$.

${ }^{1} \mathrm{H}-\mathrm{NMR}\left(\mathrm{CDCl}_{3}, 600 \mathrm{MHz}\right): \delta=10.30(\mathrm{~s}, 1 \mathrm{H}), 4.12$ (s, 3H), $4.08(\mathrm{~s}, 3 \mathrm{H})$.

${ }^{13} \mathrm{C}-\mathrm{NMR}\left(\mathrm{CDCl}_{3}, 150 \mathrm{MHz}\right): 165.44,162.87,157.71,97.83,87.19,54.54,53.98$.

MS (EI, $70 \mathrm{ev),} \mathrm{m/z} \mathrm{( \% ):} 203.0$ (40), 202.0 (76), 201.0 (100), 185.0 (22), 174.0 (17), 172.0 (43), 154.9 (17), 128.9 (10), 75.9 (23), 69.9 (10).

IR: 3008 (w), 2956 (w), 2882 (w), 2797 (w), 1686 (s), 1528 (s), 1449 (s), 1356 (s), 1324 (s), 1205 (m), 1014 (m), 789 (s).

HRMS (EI): calcd. for $\mathrm{C}_{7} \mathrm{H}_{7} \mathrm{ClN}_{2} \mathrm{O}_{3}\left[\mathrm{M}^{+}\right]$: 202.0145, found: 202. 0126.

\section{4,6-dimethoxy-1H-pyrazolo[3,4-d]pyrimidine (13).}

To a stirring solution of 4-chloro-2,6-dimethoxypyrimidine-5-carbaldehyde (12) (1.01 mg, 5 $\mathrm{mmol})$ in $\mathrm{EtOH}(80 \mathrm{~mL})$ at $\mathrm{rt}$ was added hydrazine monohydrate $(0.9 \mathrm{~g}, 15 \mathrm{mmol})$. The mixture was refluxing during $30 \mathrm{~min}$. After cooling to room temperature, the precipitate was filtered off and recrystallized in EtOH 95\% to give a white powder $\mathbf{1 3}$ (mp: > $300{ }^{\circ} \mathrm{C}$ ) in $91 \%$ yield.

${ }^{1} \mathrm{H}-\mathrm{NMR}$ (DMSO, $\left.300 \mathrm{MHz}\right): \delta=8.05$ (s, 1H), 4.04 (s, 3H), 3.93 (s, 3H).

${ }^{13}$ C-NMR (DMSO, 75 MHz): 164.44, 163.87, 157.77, 97.83, 88.19, 54.52, 53.93.

MS (EI, $70 \mathrm{ev),} \mathrm{m/z} \mathrm{( \% ):} 180.1$ (100), 179.1 (66), 1650 (12), 150.1 (36), 135.0 (67), 109.0 (13), 69.9 (11).

IR (KBr): 3252 (w), 2955 (w), 1608 (s), 1583 (m), 1503 (m), 1386 (m), 1320 (w), 1153 (m), $1081(\mathrm{w}), 942(\mathrm{~s}), 788(\mathrm{~s})$.

HRMS (EI): calcd. for $\mathrm{C}_{7} \mathrm{H}_{8} \mathrm{~N}_{4} \mathrm{O}_{4}\left[\mathrm{M}^{+}\right]$: 180.0647 , found: 180.0643 .

\section{$1 H$-pyrazolo[3,4-d]pyrimidine-4,6(5H,7H)-dione, oxypurinol (2).}

A mixture of 4,6-dimethoxy-1H-pyrazolo[3,4-d]pyrimidine (13) (0.54 g, 3mmol) with concentrated hydrochloric acid $(30 \mathrm{~mL})$ was heated under reflux for $1 \mathrm{~h}$. After the reaction was complete, the solution was treated with activated charcoal and evaporated under reduced pressure. The residue was recrystallized from water to afford $1.60 \mathrm{~g}$ of oxypurinol (2) (81\% yield) as a white solid (mp: $>300{ }^{\circ} \mathrm{C}$ ).

${ }^{1} \mathrm{H}-\mathrm{NMR}$ (DMSO, $300 \mathrm{MHz}$ ): $\delta=13.27$ (s, 1H), 11.30 (s, 1H), 10.63 (s, 1H), 8.33 (s, 1H).

${ }^{13} \mathrm{C}-\mathrm{NMR}$ (DMSO, $75 \mathrm{MHz}$ ): 159.60, 151.62, 150.78, 128.93, 100.20. 
MS (EI, $70 \mathrm{ev}), \mathrm{m} / z(\%): 152.0$ (100), 109.0 (92), 52.0 (31), 44.0 (14).

IR (KBr): 3268 (m), 3124 (w), 3028 (m), 2804 (w), 1709(s), 1677 (s), 1616 (m), 1419 (m), $1244(\mathrm{~m}), 1169$ (s), 1025 (m), $810(\mathrm{~m}), 748$ (s), 700 (s).

HRMS (EI): calcd. for $\mathrm{C}_{5} \mathrm{H}_{4} \mathrm{~N}_{4} \mathrm{O}_{2}\left[\mathrm{M}^{+}\right]$: 152.0334 , found: 152.0324 .

\section{Products 14-16.}

\section{4-allyl-2,6-dimethoxy-5-(trimethylsilyl)pyrimidine (14).}

A dry and argon flushed $10 \mathrm{~mL}$ flask, equipped with a magnetic stirrer and a septum, was charged with a solution of 4-bromo-2,6-dimethoxy-5-(trimethylsilyl)pyrimidine (9a) (291 mg, $1.0 \mathrm{mmol})$ in dry THF (2 mL). $i$-PrMgCl$\cdot \mathrm{LiCl}(1.0 \mathrm{M} / \mathrm{THF}, 1.05 \mathrm{mmol}, 1.05$ equiv) was added very slowly (within $5 \mathrm{~min}$ ) at $-15^{\circ} \mathrm{C}$ and the resulting mixture was stirred for $12 \mathrm{~h}$ at $15{ }^{\circ} \mathrm{C}$ to complete the bromine-magnesium exchange (checked by GC-MS analysis of reaction aliquots). Then, allyl bromide (145 mg, $1.2 \mathrm{mmol}, 1.2$ equiv) was added dropwise. After $30 \mathrm{~min}, 3$ drops of $\mathrm{CuCN} \cdot 2 \mathrm{LiCl}$ (cat., $1 \mathrm{M}$ in THF) were added. The mixture was warmed up to $\mathrm{rt}$ for $6 \mathrm{~h}$ and was quenched with saturated aqueous $\mathrm{NH}_{4} \mathrm{Cl}$ solution. The aqueous phase was extracted with ethyl acetate $(3 \times 5 \mathrm{~mL})$. The organic fractions were dried $\left(\mathrm{Na}_{2} \mathrm{SO}_{4}\right)$ and concentrated in vacuo. Purification by flash chromatography (n-pentane/diethyl ether $=3: 2)$ yielded $204 \mathrm{mg}$ ( $81 \%$ yield $)$ of $\mathbf{1 4}$ as colorless oil.

${ }^{1} \mathrm{H}-\mathrm{NMR}\left(\mathrm{CDCl}_{3}, 600 \mathrm{MHz}\right): \delta=6.03(\mathrm{~m}, 0.8 \mathrm{H}), 5.08(\mathrm{dd}, J=9.5 \mathrm{~Hz}, 0.8 \mathrm{H}), 5.02(\mathrm{dd}, J=$ $17.1 \mathrm{~Hz}, 0.8 \mathrm{H}), 3.96-3.93(2 \mathrm{~s}, 6 \mathrm{H}), 3.47$ (d, $J=6.2 \mathrm{~Hz}, 2 \mathrm{H}), 0.31(\mathrm{~s}, 9 \mathrm{H})$.

${ }^{13} \mathrm{C}-\mathrm{NMR}\left(\mathrm{CDCl}_{3}, 150 \mathrm{MHz}\right): 176.02,175.29,135.79,116.2,107.05,54.27,53.40,42.07$, $29.69,1.41$.

MS (EI, $70 \mathrm{ev),} \mathrm{m/z} \mathrm{( \% ):} 253.1$ (11), 252.1 (56), 251.1 (100), 237.1 (19), 207.1 (23), 89.0 (25).

IR (film): 3081 (w), 2980 (w), 2960 (w), 2870 (w), 1638 (w), 1571 (s),1457 (m), 1370 (s), 1362 (s), $1210(\mathrm{~m}), 1084(\mathrm{w})$.

HRMS (EI): calcd. for $\mathrm{C}_{12} \mathrm{H}_{20} \mathrm{~N}_{2} \mathrm{O}_{2} \mathrm{Si}\left[\mathrm{M}^{+}\right]$: 252.1294, found: 252.1266 .

\section{(5-(cyclohexyl(hydroxy)methyl)-2,6-dimethoxypyrimidin-4-yl)(morpholino)methanone} (15).

A dry and argon flushed $10 \mathrm{~mL}$ flask, equipped with a magnetic stirrer and a septum, was charged with a solution of 4,5-dibromo-2,6-dimethoxypyrimidine (5) (596 mg, 2 mmol) in dry THF (3 mL). $i$-PrMgCl-LiCl (1.0 M/THF, $2.1 \mathrm{mmol}, 1.05$ equiv) was added very slowly 
(within $5 \mathrm{~min}$ ) at room temperature and the resulting mixture was stirred for $15 \mathrm{~min}$ to complete the bromine-magnesium exchange (checked by GC-MS analysis of reaction aliquots). Cyclohexanecarboxaldehyde ( $266 \mathrm{mg}, 2.2 \mathrm{mmol}, 1.2$ equiv) was added at $\mathrm{rt}$ and the mixture was stirred at $\mathrm{rt}$ for 12 h. $i$-PrMgCl-LiCl (1.0 M/THF, $2.0 \mathrm{mmol}, 1.5$ equiv) was added at $\mathrm{rt}$ to the mixture which was stirred for $24 \mathrm{~h}$ to complete the second bromine-magnesium exchange (checked by GC-MS analysis of reaction aliquots). 4-morpholinecarbonyl chloride (597 mg, 2 equiv) was added and the resulting mixture was stirred at $\mathrm{rt}$ for $12 \mathrm{~h}$. Then, the mixture was quenched with saturated aqueous $\mathrm{NH}_{4} \mathrm{Cl}$ solution. The aqueous phase was extracted with ethyl acetate $(3 \times 10 \mathrm{~mL})$. The organic fractions were dried $\left(\mathrm{Na}_{2} \mathrm{SO}_{4}\right)$ and concentrated in vacuo. Purification by flash chromatography (diethyl ether) yielded $503 \mathrm{mg}$ (69\% yield) of $\mathbf{1 5}$ as a colorless oil.

${ }^{1} \mathrm{H}-\mathrm{NMR}\left(\mathrm{CDCl}_{3}, 300 \mathrm{MHz}\right): \delta=8.09$ (s, 1H), 5.64 (d, J = 6.7 Hz), 3.99 (s, 3H), 3.97 (s, 3H), $1.88-0.88(\mathrm{~m}, 11 \mathrm{H})$.

${ }^{13} \mathrm{C}-\mathrm{NMR}\left(\mathrm{CDCl}_{3}, 75 \mathrm{MHz}\right): 168.34,164.63,156.24,154.62,114.15,74.04,66.57,54.05$, $44.14,42.13,28.84,28.40$.

MS (EI, $70 \mathrm{ev),} \mathrm{m/z} \mathrm{( \% ):} 365.3$ (1), 282.2 (2), 235.2 (24), 153.1 (100), 114.1 (12), 70.1 (6).

IR (film): 2924 (m), 2852 (m), 1700 (s), 1599 (m), 1567 (m), 1399 (m),1218 (s), 1069 (m).

HRMS (EI): calcd. for $\mathrm{C}_{18} \mathrm{H}_{27} \mathrm{~N}_{3} \mathrm{O}_{5}\left[\mathrm{M}^{+}\right]$: 365.1951, found: 365.1935 .

\section{5-allyl-2,4-dimethoxy-6-methylpyrimidine (16).}

A dry and argon flushed $10 \mathrm{~mL}$ flask, equipped with a magnetic stirrer and a septum, was charged with a solution of 4,5-dibromo-2,6-dimethoxypyrimidine (5) (596 mg, $2 \mathrm{mmol}$ ) in dry THF (3 mL). $i$-PrMgCl-LiCl (1.0 M/THF, $2.1 \mathrm{mmol}$, 1.05 equiv) was added very slowly (within $5 \mathrm{~min}$ ) at room temperature and the resulting mixture was stirred for $15 \mathrm{~min}$ to complete the bromine-magnesium exchange (checked by GC-MS analysis of reaction aliquots). Allyl bromide ( $266 \mathrm{mg}, 2.2 \mathrm{mmol}, 1.1$ equiv) was added dropwise. The mixture was stirred at $\mathrm{rt}$ for $2 \mathrm{~h}$. Then, the mixture was cooled down to $-5{ }^{\circ} \mathrm{C}$ and $i$-PrMgCl$\cdot \mathrm{LiCl}(1.0$ M/THF, $3.0 \mathrm{mmol}$, 1.5 equiv) was added slowly. After $8 \mathrm{~h}$, the Br/Mg-exchange was complete (checked by GC-MS analysis of reaction aliquots) and methylene iodide ( $850 \mathrm{mg}, 3$ equiv) was added to the mixture at $-5{ }^{\circ} \mathrm{C}$ following by the addition of 3 drops of $\mathrm{CuCN} \cdot 2 \mathrm{LiCl}$ (cat., $1 \mathrm{M}$ in $\mathrm{THF}$ ). The mixture was warmed up to room temperature for $4 \mathrm{~h}$ and quenched with saturated aqueous $\mathrm{NH}_{4} \mathrm{Cl}$ solution. The aqueous phase was extracted with ethyl acetate $(3 \times 10 \mathrm{~mL})$. The organic fractions were dried $\left(\mathrm{Na}_{2} \mathrm{SO}_{4}\right)$ and concentrated in vacuo. 
Purification by flash chromatography (n-pentane/diethyl ether $=4: 1)$ yielded $408 \mathrm{mg}(81 \%$ yield) of $\mathbf{1 6}$ as a colorless oil.

${ }^{1} \mathrm{H}-\mathrm{NMR}\left(\mathrm{CDCl}_{3}, 400 \mathrm{MHz}\right): \delta=5.8(\mathrm{~m}, 1 \mathrm{H}), 4.97(\mathrm{dq}, J=10.2 \mathrm{~Hz}, 1 \mathrm{H}), 4.90(\mathrm{dq}, J=17.1$ $\mathrm{Hz}, 1 \mathrm{H}), 3.94$ (s, 6H), 3.25 (dt, $J=5.9 \mathrm{~Hz}, 2 \mathrm{H}), 2.33$ (s, 3H).

${ }^{13} \mathrm{C}-\mathrm{NMR}\left(\mathrm{CDCl}_{3}, 100 \mathrm{MHz}\right): 169.27,166.60,163.0,134.75,114.92,109.71,54.33,53.89$, 28.67, 21.23.

MS (EI, $70 \mathrm{ev),} \mathrm{m/z} \mathrm{( \% ):} 196.1$ (45), 194.1 (100), 193.1 (49), 179.1 (28), 164.1 (29), 138.0 (11), 122.1 (5), 94.1 (6), 56.0 (21).

IR (film): 2980 (w), 2955 (w), 2871 (w), 1638 (w), 1571 (s), 1457 (m), 1369 (s), 1358 (s), 1203 (m), 1090 (w).

HRMS (EI): calcd. for $\mathrm{C}_{10} \mathrm{H}_{14} \mathrm{~N}_{2} \mathrm{O}_{2}\left[\mathrm{M}^{+}\right]$: 194.1055, found: 194.1036 .

\section{Synthesis of emivirine (3).}

\section{2-(4-bromo-2,6-dimethoxypyrimidin-5-yl)propan-2-ol (17).}

In a flame dried, argon-flushed $50 \mathrm{~mL}$ Schlenk-flask equipped with a septum and a magnetic stirring bar was placed $\mathrm{LaCl}_{3} \cdot 2 \mathrm{LiCl}$ in THF $(0.33 \mathrm{M} ; 30 \mathrm{~mL}, 10.00 \mathrm{mmol}, 1.00$ equiv). Acetone $(580 \mathrm{mg}, 10.0 \mathrm{mmol})$ was added and the resulting mixture was stirred for $2 \mathrm{~h}$ at $\mathrm{rt}$. A second dry and argon flushed $25 \mathrm{~mL}$ flask, equipped with a magnetic stirrer and a septum, was charged with a solution of 4,5-dibromo-2,6-dimethoxypyrimidine (5) (2.98 g, $10 \mathrm{mmol})$ in dry THF (10 mL). $i$-PrMgCl. $\mathrm{LiCl}(1.0 \mathrm{M} / \mathrm{THF}, 10.5 \mathrm{mmol}, 1.05$ equiv) was added very slowly (within $5 \mathrm{~min}$ ) at room temperature and the resulting mixture was stirred for $15 \mathrm{~min}$ to complete the bromine-magnesium exchange (checked by GC-MS analysis of reaction aliquots) and obtain the Grignard reagent 7. The mixture of acetone with $\mathrm{LaCl}_{3} \cdot 2 \mathrm{LiCl}$ in $\mathrm{THF}$ was cooled to $0{ }^{\circ} \mathrm{C}$ and the solution of the Grignard reagent 7 was added dropwise. The resulting mixture was allowed to stir at the same temperature for $4 \mathrm{~h}$ and was quenched with sat. aq. $\mathrm{NH}_{4} \mathrm{Cl}(20 \mathrm{~mL})$ and water $(20 \mathrm{~mL})$ was added. The aqueous layer was extracted with ethyl acetate $(4 \times 30 \mathrm{~mL})$, the combined extracts were dried $\left(\mathrm{Na}_{2} \mathrm{SO}_{4}\right)$ and evaporated in vacuo. The crude residue was purified by flash column chromatography (n-pentane/diethyl ether $=4: 1)$ yielded $2.40 \mathrm{~g}(81 \%$ yield $)$ of $\mathbf{1 7}$ as a white oil.

${ }^{1} \mathrm{H}-\mathrm{NMR}\left(\mathrm{CDCl}_{3}, 400 \mathrm{MHz}\right): \delta=4.03(\mathrm{~s}, 3 \mathrm{H}), 3.97$ (s, 3H), $3.95(\mathrm{~s}, 3 \mathrm{H}), 1.70(\mathrm{~s}, 6 \mathrm{H})$.

${ }^{13} \mathrm{C}-\mathrm{NMR}\left(\mathrm{CDCl}_{3}, 150 \mathrm{MHz}\right):$ 168.97, 161.17, 149.00, 120.74, 72.44, 55.24, 54.99, 30.17.

MS (EI, 70 ev), m/z (\%): 279.0 (3), 277.0 (3), 262.9 (100), 260.9 (97), 244.9 (29), 220.9 (5), 167.0 (7). 
IR (film): 3563 (m), 2950 (w), 1562 (m), 1527 (s), 1481 (m), 1383 (m), 1352 (m), 1215 (m), 1013 (s).

HRMS (EI): calcd. for $\mathrm{C}_{9} \mathrm{H}_{13} \mathrm{BrN}_{2} \mathrm{O}_{3}[\mathrm{M}+\mathrm{H}]^{+}: 277.0110$, found[M+H] $]^{+}: 277.0200$.

\section{4-bromo-5-isopropyl-2,6-dimethoxypyrimidine (18).}

To an ice-cooled, rapidly stirred slurry of 17 (1.38 g, 5mmol) dissolved in dry dichloromethane $(20 \mathrm{~mL})$ in triethylsilane $(1.74 \mathrm{~g}, 3$ equiv) was slowly added trifluoroacetic acid (1.99 g, 3.5 equiv). When the addition was complete, the mixture was warmed to room temperature and stirred overnight $(12 \mathrm{~h})$. At the end of this period, the mixture was poured into $30 \mathrm{~mL}$ of saturated $\mathrm{NaHCO}_{3}$ and extracted with dichloromethane $(3 \times 20 \mathrm{~mL})$. Extracts were combinated, dried over $\mathrm{Na}_{2} \mathrm{SO}_{4}$, filtered, and concentrated under reduced pressure at 40 ${ }^{\circ} \mathrm{C}$ (oil bath) to give $1.28 \mathrm{~g}$ (95\%) of a colorless oil. The product was identified as a mixture of 4-bromo-5-isopropyl-2,6-dimethoxypyrimidine $\mathbf{1 8}(17 \%)$ and the corresponding dehydrated product 18a (83\%) (4-bromo-2,6-dimethoxy-5-(prop-1-en-2-yl)pyrimidine). The mixture of $\mathbf{1 8}$ and $\mathbf{1 8 a}$ was used for the next step without purification.

${ }^{1} \mathrm{H}-\mathrm{NMR}\left(\mathrm{CDCl}_{3}, 200 \mathrm{MHz}\right): \delta=5.34(\mathrm{~s}, 1.0 \mathrm{H}), 4.95(\mathrm{t}, 1.0 \mathrm{H}), 3.95(\mathrm{~m}, 7.1 \mathrm{H}), 3.40(\mathrm{q}$, $0.37 \mathrm{H}), 1.96(\mathrm{~m}, 3.03 \mathrm{H}) *, 1.26-1.23(\mathrm{~d}, 1.17 \mathrm{H}) *$.<smiles>C=C(C)c1c(Br)nc(OC)nc1OC</smiles>

18a: dehydrated product from 18

*These integrations give respectively the yield of $\mathbf{1 8 a}(83 \%)$ and $\mathbf{1 8}(17 \%)$.

The mixture of $\mathbf{1 8}$ and 18a $(1.27 \mathrm{~g})$ is directly used and solved in dry ethanol $(10 \mathrm{~mL})$. Platinium oxide $(87 \mathrm{mg})$ is added to the solution and the resulting mixture is stirred at atmospheric pressure in a hydrogen atmosphere for $30 \mathrm{~min}$. The catalyst was removed by filtration and the filtrate was concentrated in vacuo. The crude residue was purified by flash column chromatography (n-pentane/diethyl ether $=4: 1)$ yielded $1.17 \mathrm{~g}(91 \%)$ of $\mathbf{1 8}$ as a colorless oil.

${ }^{1} \mathrm{H}-\mathrm{NMR}\left(\mathrm{CDCl}_{3}, 400 \mathrm{MHz}\right): \delta=3.98$ (s, 3H), 3.95 (s, 3H), 3.39 (septuplet, ${ }^{3} J=7.1 \mathrm{~Hz}$ ), 1.24 $\left(\mathrm{d},{ }^{3} J=7.0 \mathrm{~Hz}, 6 \mathrm{H}\right)$.

${ }^{13} \mathrm{C}-\mathrm{NMR}\left(\mathrm{CDCl}_{3}, 150 \mathrm{MHz}\right): 169.49,161.74,152.23,120.21,55.07,54.27,30.47,19.68$. MS (EI, $70 \mathrm{ev),} \mathrm{m/z} \mathrm{( \% ):} 262.0$ (13), 260.0 (13), 246.9 (95), 244.9 (100), 165.0 (8), 151.0 (6). 
IR (film): 2986 (w), 2959 (w), 2874 (w), 1575 (m), 1534 (s), 1451 (m), 1362 (s), 1215 (m), 1017 (s).

HRMS (EI): calcd. for $\mathrm{C}_{9} \mathrm{H}_{13} \mathrm{BrN}_{2} \mathrm{O}_{2}[\mathrm{M}+]$ : 260.0160, found[M+]: 260.0171 .

\section{4-benzyl-5-isopropyl-2,6-dimethoxypyrimidine (19).}

A dry and argon flushed $10 \mathrm{~mL}$ flask, equipped with a magnetic stirrer and a septum, was charged with a solution of 4-bromo-5-isopropyl-2,6-dimethoxypyrimidine (18) (783 mg, 3.0 $\mathrm{mmol})$ in dry THF (2 mL). $i$-PrMgCl$\cdot \mathrm{LiCl}(1.0 \mathrm{M} / \mathrm{THF}, 3.15 \mathrm{mmol}, 1.05$ equiv) was added at $\mathrm{rt}$ and the resulting mixture was stirred for $5 \mathrm{~h}$ at this temperature to complete the brominemagnesium exchange (checked by GC-MS analysis of reaction aliquots). Then, benzyl bromide (1.03 g, $6 \mathrm{mmol}, 2$ equiv) was added dropwise. The mixture was stirred for $20 \mathrm{~h}$ and was quenched with saturated aqueous $\mathrm{NH}_{4} \mathrm{Cl}$ solution. The aqueous phase was extracted with ethyl acetate $(3 \times 10 \mathrm{~mL})$. The organic fractions were dried $\left(\mathrm{Na}_{2} \mathrm{SO}_{4}\right)$ and concentrated in vасио. Purification by flash chromatography (n-pentane/diethyl ether $=1: 1$ ) yielded $709 \mathrm{mg}$ ( $87 \%$ yield) of $\mathbf{1 9}$ as a pale yellow oil.

${ }^{1} \mathrm{H}-\mathrm{NMR}\left(\mathrm{CDCl}_{3}, 400 \mathrm{MHz}\right): \delta=7.22(\mathrm{~m}, 5 \mathrm{H}), 4.06$ (s, 2H), 3.95 (2 singulets, $\left.6 \mathrm{H}\right), 3.16$ (septuplet, $\left.{ }^{3} J=7.0 \mathrm{~Hz}, 1 \mathrm{H}\right), 1.11\left(\mathrm{~d},{ }^{3} J=7.0 \mathrm{~Hz}, 6 \mathrm{H}\right)$.

${ }^{13} \mathrm{C}-\mathrm{NMR}\left(\mathrm{CDCl}_{3}, 150 \mathrm{MHz}\right): 170.25,166.28,162.46,138.77,126.53,128.32,126.17$, $117.82,54.36,53.42,41.43,26.73,20.10$.

MS (EI, $70 \mathrm{ev),} \mathrm{m/z} \mathrm{( \% ):} 272.1$ (80), 271.1 (100), 257.1 (93), 244.1 (29), 243.1 (23), 241.1 (16), 227.0 (10), 167.1 (14), 91.1 (14).

IR (film): 2956 (w), 2872 (w), 1562 (s), 1475 (w), 1451 (m), 1366 (s), 1217 (m), 1035 (w). HRMS (EI): calcd. for $\mathrm{C}_{16} \mathrm{H}_{20} \mathrm{~N}_{2} \mathrm{O}_{2}[\mathrm{M}+]$ : 272.1525, found[M+]: 272.1534 .

\section{6-benzyl-5-isopropylpyrimidine-2,4(1H,3H)-dione (20).}

A solution of 4-benzyl-5-isopropyl-2,6-dimethoxypyrimidine (19) (0.544 g, 2mmol) in $\mathrm{MeOH}$ $(10 \mathrm{~mL})$ with concentrated hydrochloric acid $(30 \mathrm{~mL})$ was heated under reflux for $4 \mathrm{~h}$. After the reaction was complete, the solution was treated with activated charcoal and evaporated under reduced pressure. The residue was recrystallized from $\mathrm{MeOH} / \mathrm{H}_{2} \mathrm{O}(4 / 1)$ to afford 450 mg (20) (92\% yield) as a white solid (mp: 103.1.-104.2).

${ }^{1} \mathrm{H}-\mathrm{NMR}\left(\mathrm{CDCl}_{3}, 400 \mathrm{MHz}\right): \delta=8.18$ (s, 1H), 7.94 (s, 1H), 7.38-7.29 (m, 3H), 7.19 (dd, 2H), 3.82 (s, 2H), 3.03 (septuplet, $\left.{ }^{3} J=7.0 \mathrm{~Hz}, 1 \mathrm{H}\right), 1.30$ (d, $\left.{ }^{3} J=7.0 \mathrm{~Hz}, 6 \mathrm{H}\right)$.

${ }^{13} \mathrm{C}-\mathrm{NMR}\left(\mathrm{CDCl}_{3}, 150 \mathrm{MHz}\right): 163.73,150.81,148.28,136.95,128.50,127.87,126.51$, $113.81,35.15,26.28,20.00$. 
MS (EI, $70 \mathrm{ev),} \mathrm{m/z} \mathrm{( \% ):} 244.1$ (72), 230.1 (17), 215.0 (5), 229.1 (100), 186.0 (14), 153.0 (7), 91.0 (17), 69.0 (7).

IR (KBr): 2957 (w), 2869 (w), 1724 (s), 1640 (S), 1461 (m), 1411 (w), 1358 (w), 1193 (w).

HRMS (EI): calcd. for $\mathrm{C}_{14} \mathrm{H}_{16} \mathrm{~N}_{2} \mathrm{O}_{2}[\mathrm{M}+]$ : 244.1212, found[M+]: 244.1225.

\section{6-benzyl-1-(ethoxymethyl)-5-isopropylpyrimidine-2,4(1H,3H)-dione, emivirine (3).}

6-benzyl-5-isopropylpyrimidine-2,4(1H,3H)-dione (20) (366 mg, $1.5 \mathrm{mmol})$ was suspended in dry acetonitrile $(10 \mathrm{~mL})$ under nitrogen and BSA $(1.33 \mathrm{ml}, 5.2 \mathrm{mmol})$ was added. The reaction mixture was stirred for $10 \mathrm{~min}$ at $\mathrm{rt}$ and then cooled to $-45^{\circ} \mathrm{C}$. Diethoxymethane $(218$ $\mathrm{mg}, 3 \mathrm{mmol})$ in dry acetonitrile $(1 \mathrm{~mL})$ and TMS-triflate $(350 \mathrm{mg}, 1.6 \mathrm{mmol})$ in dry acetonitrile $(1 \mathrm{~mL})$ were added to the reaction mixture, which was slowly warmed to $\mathrm{rt}$ for 3.5 h. The reaction mixture was quenched with saturated aqueous NaHCO3 (10 mL) followed by evaporation under reduced pressure. Water $(20 \mathrm{~mL})$ was added followed by the extraction with diethyl acetate $(3 \times 20 \mathrm{~mL})$. The combinated organic phases were dried and evaporated under reduce pressure. Purification by recristallization from EtOH/ $\mathrm{H}_{2} \mathrm{O}(4 / 1)$ to afford 407 mg (3) (90\% yield) as a white solid (mp: 109.1-110.7).

${ }^{1} \mathrm{H}-\mathrm{NMR}\left(\mathrm{CDCl}_{3}, 400 \mathrm{MHz}\right): \delta=9.2(\mathrm{~s}, 1 \mathrm{H}), 7.34-7.27$ (m, 3H), 7.11-7.12 (m, 2H), 5.12 (s, 2H), 4.18 (s, 2H), 3.62 (q, $\left.{ }^{3} J=6.9 \mathrm{~Hz}, 2 \mathrm{H}\right), 2.86$ (septuplet, $\left.{ }^{3} J=6.9 \mathrm{~Hz}, 1 \mathrm{H}\right), 1.28\left(\mathrm{~d},{ }^{3} J=6.9\right.$ $\mathrm{Hz}, 6 \mathrm{H}), 1.18(\mathrm{t}, J=6.9 \mathrm{~Hz}, 3 \mathrm{H})$.

${ }^{13} \mathrm{C}-\mathrm{NMR}\left(\mathrm{CDCl}_{3}, 150 \mathrm{MHz}\right): 162.33,151.87,148.56,135.35,129.17,127.23,127.20$, $119.69,72.88,65.00,33.46,28.33,20.37,15.03$.

MS (EI, $70 \mathrm{ev),} \mathrm{m/z} \mathrm{( \% ):} 303.1$ (19), 302.1 (60), 287.1 (16), 273.1 (39), 257.1 (100), 241.1 (80), 229.1 (64), 135.1 (34), 91.0 (21), 59.0 (29).

IR (KBr): 3188 (w), 2968 (w), 1710 (s), 1673 (s), 1445 (m), 1100 (m), 1025 (m), 719 (m). HRMS (EI): calcd. for $\mathrm{C}_{17} \mathrm{H}_{22} \mathrm{~N}_{2} \mathrm{O}_{3}[\mathrm{M}+]$ : 302.1630, found[M+]: 302.1641 . 
Spectrum:<smiles>COc1nc(Cl)c(Br)c(OC)n1</smiles>
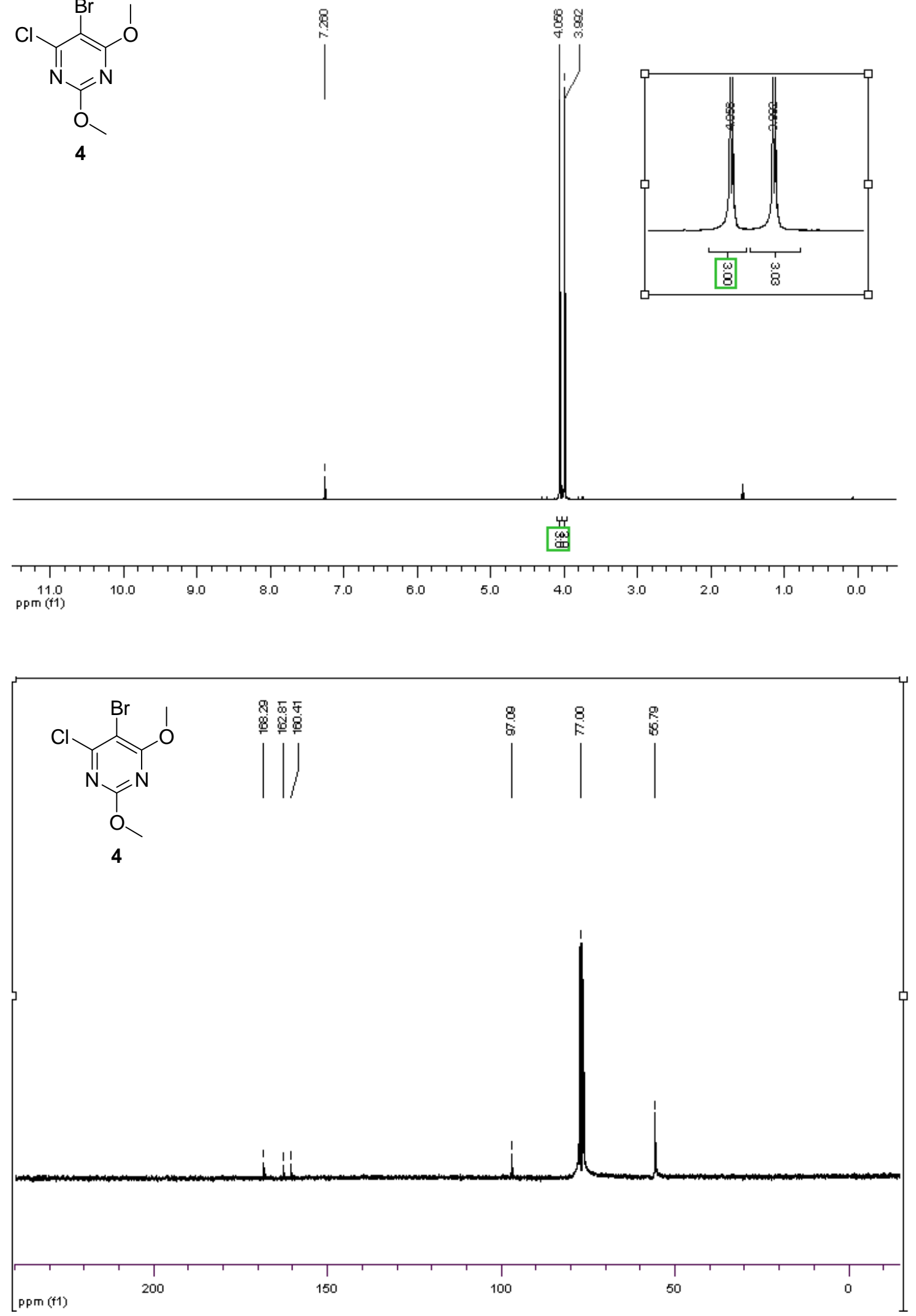
4-bromo -2,6-dimethoxypyrimidine.
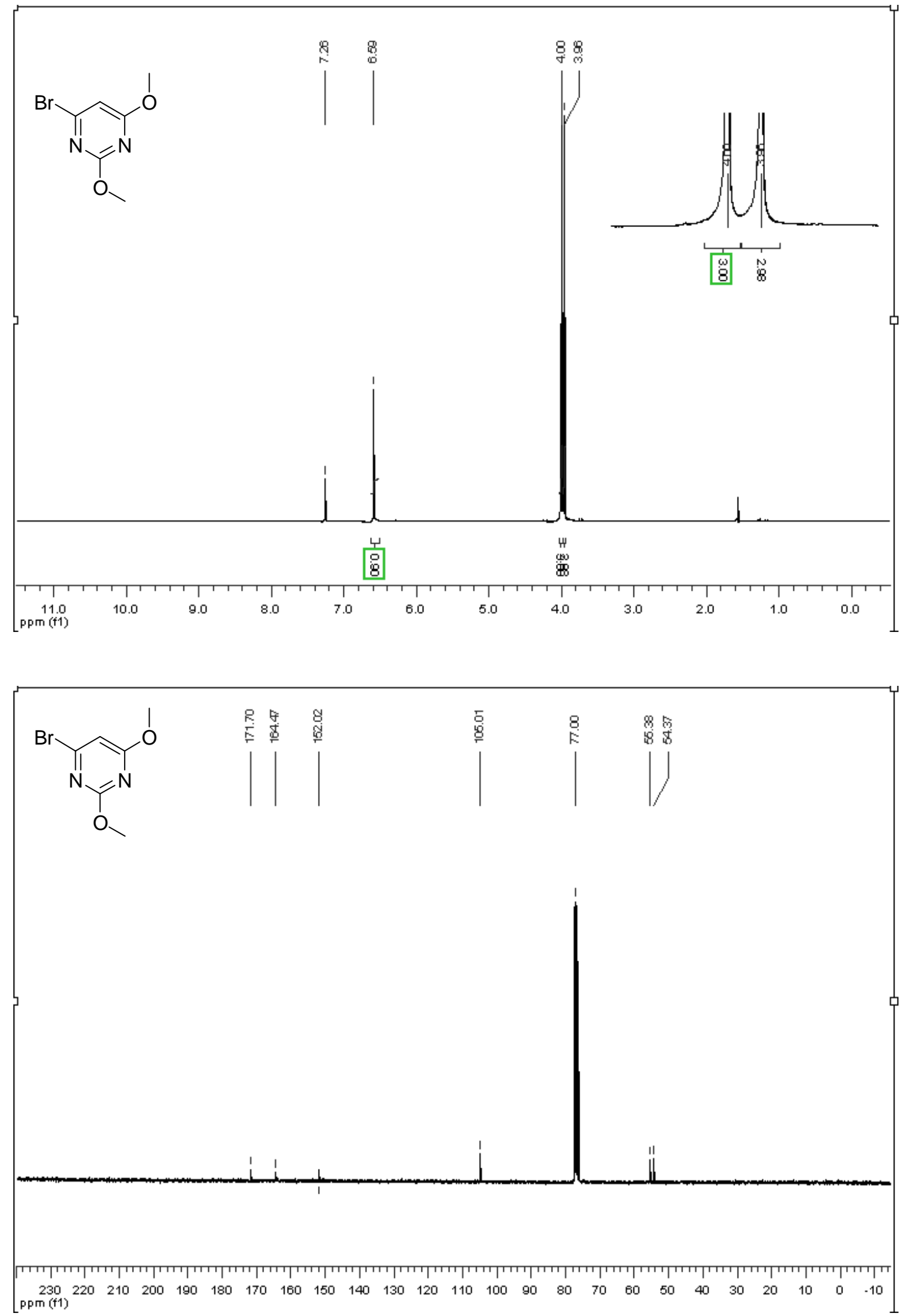

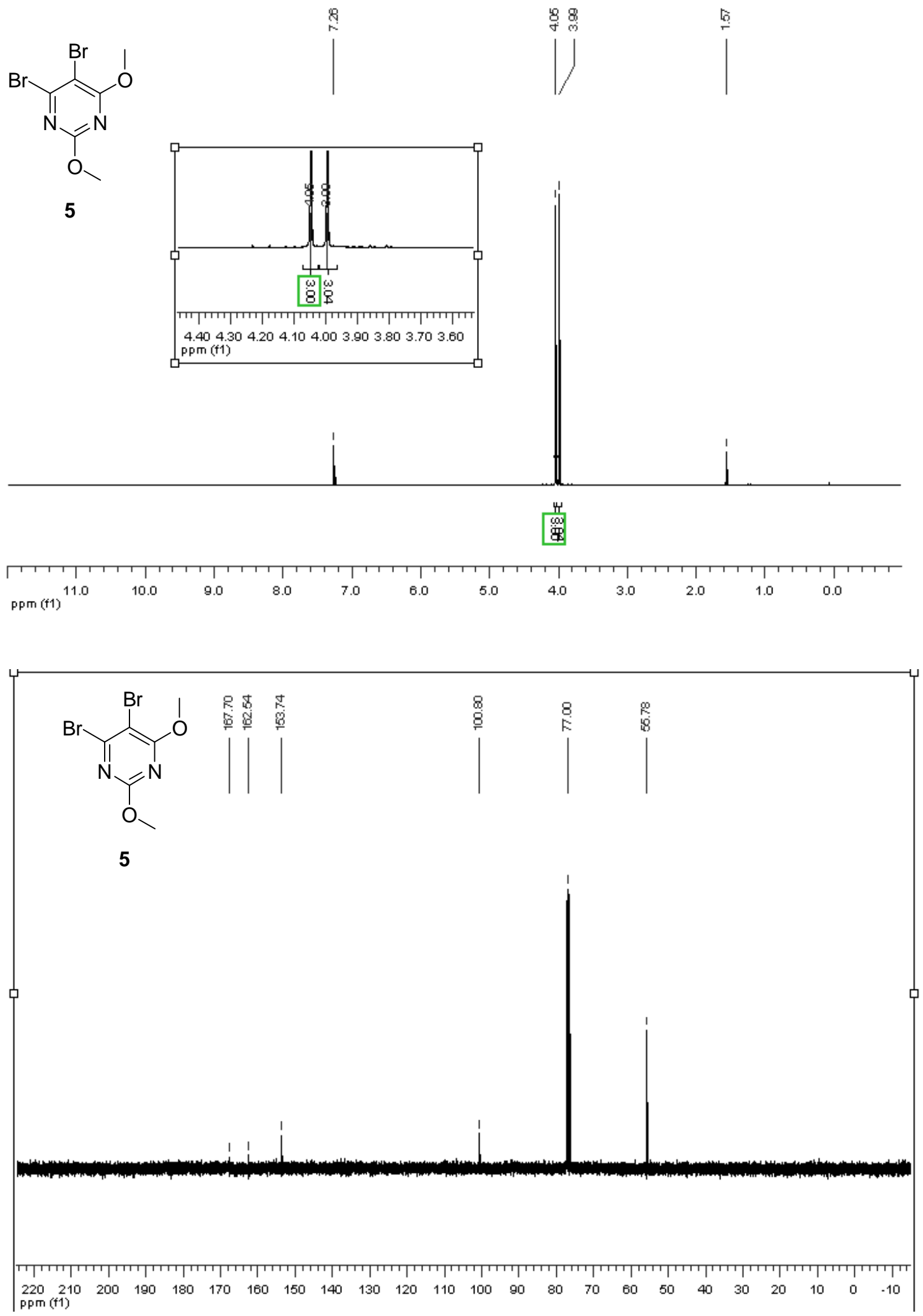

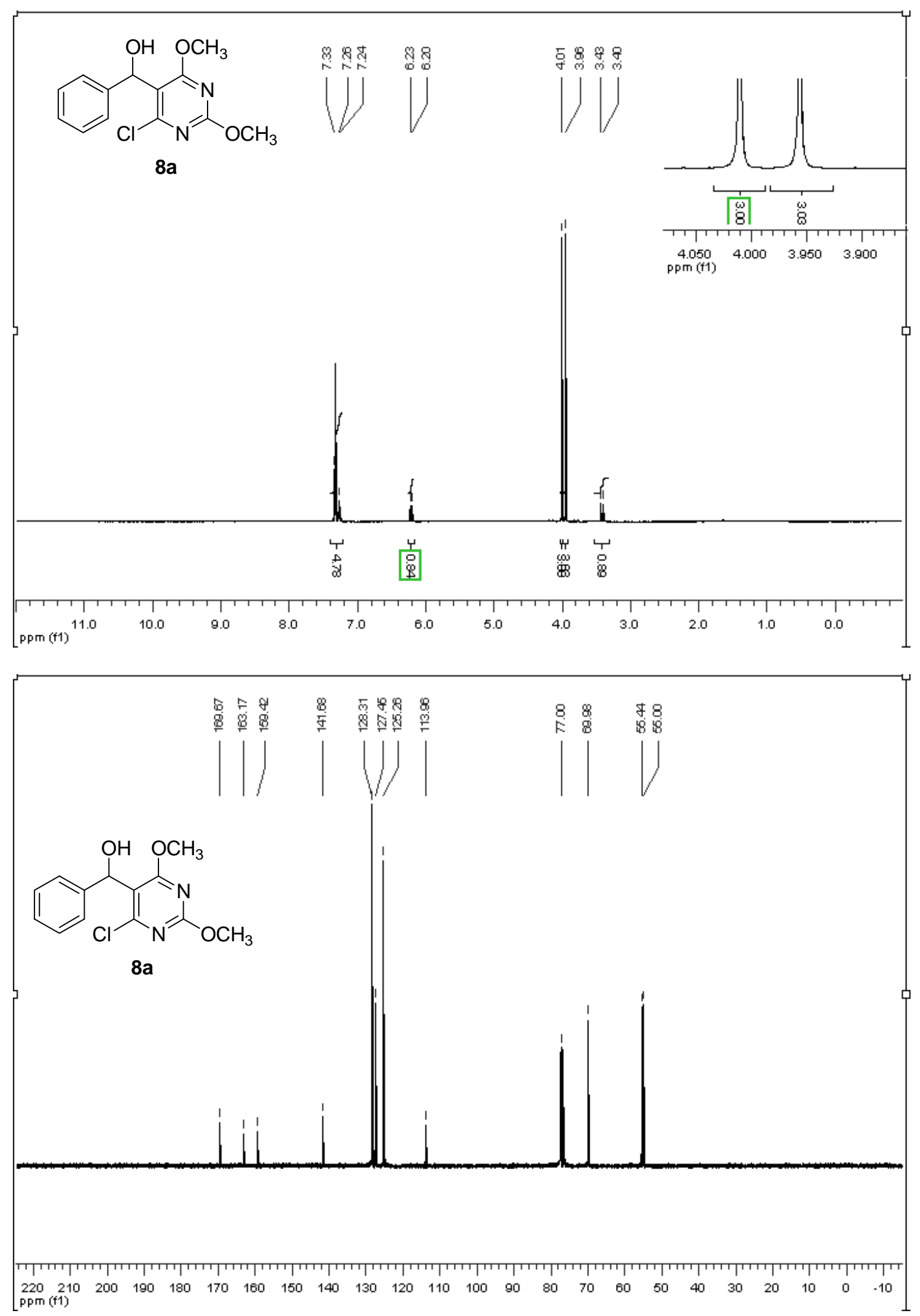


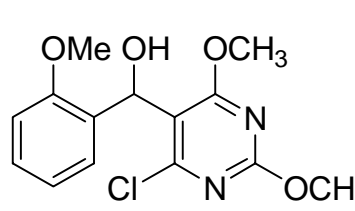

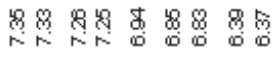
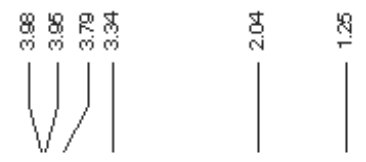

$8 b$

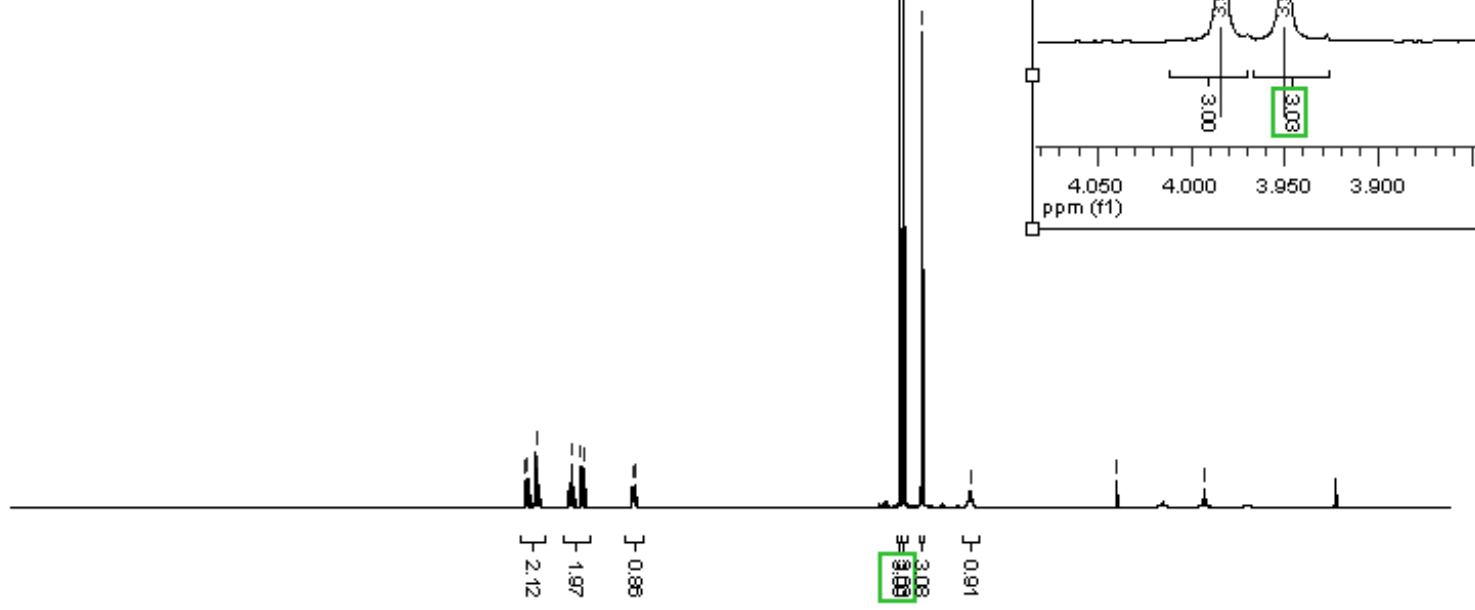

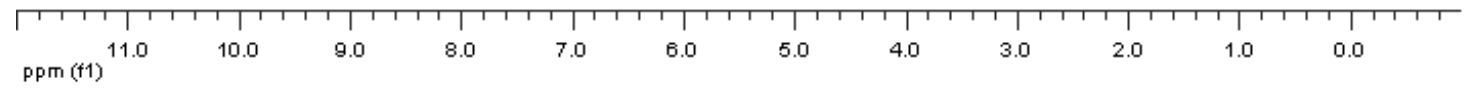
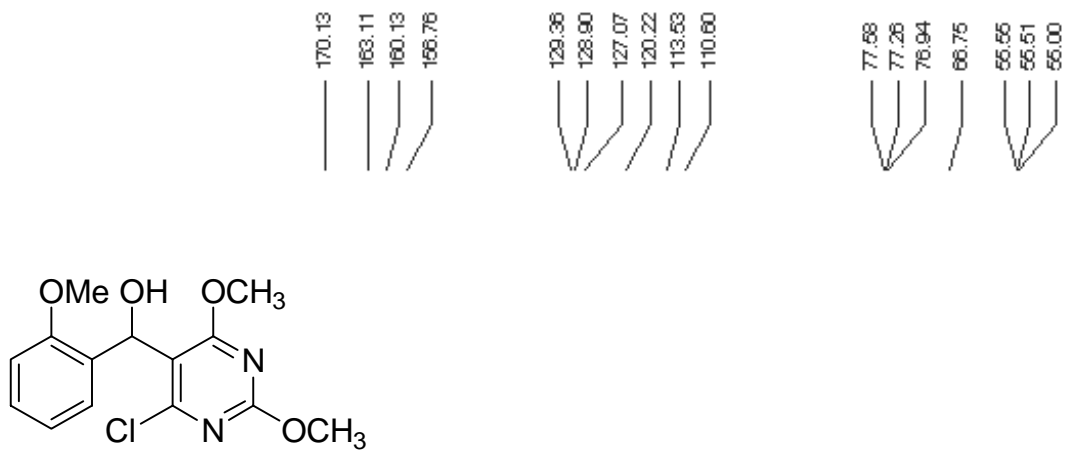

$8 b$

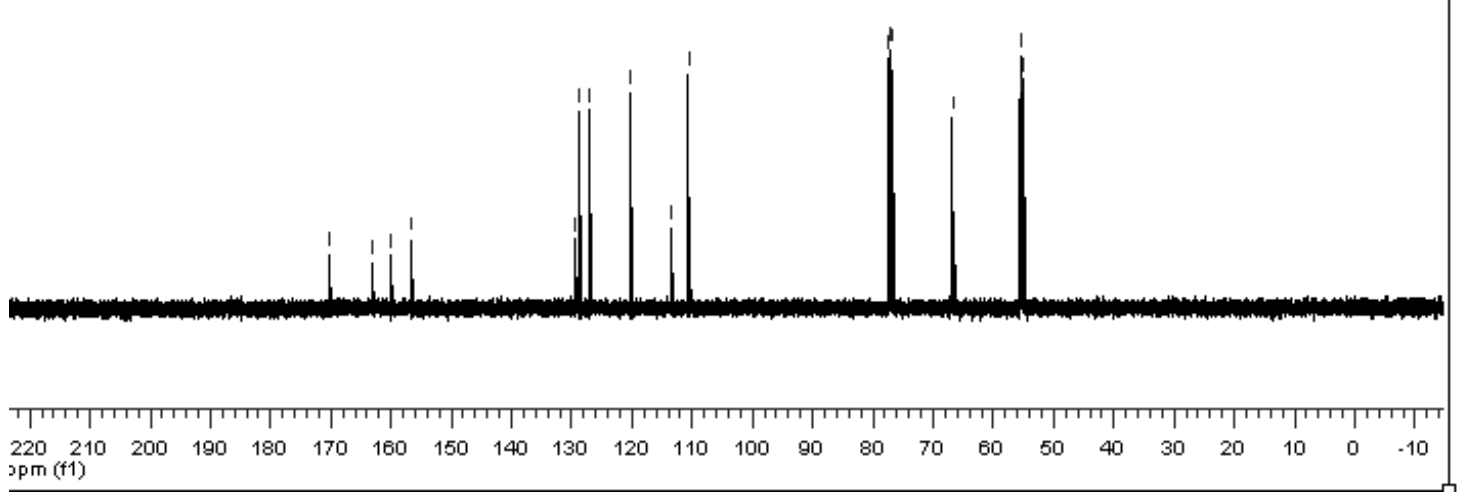



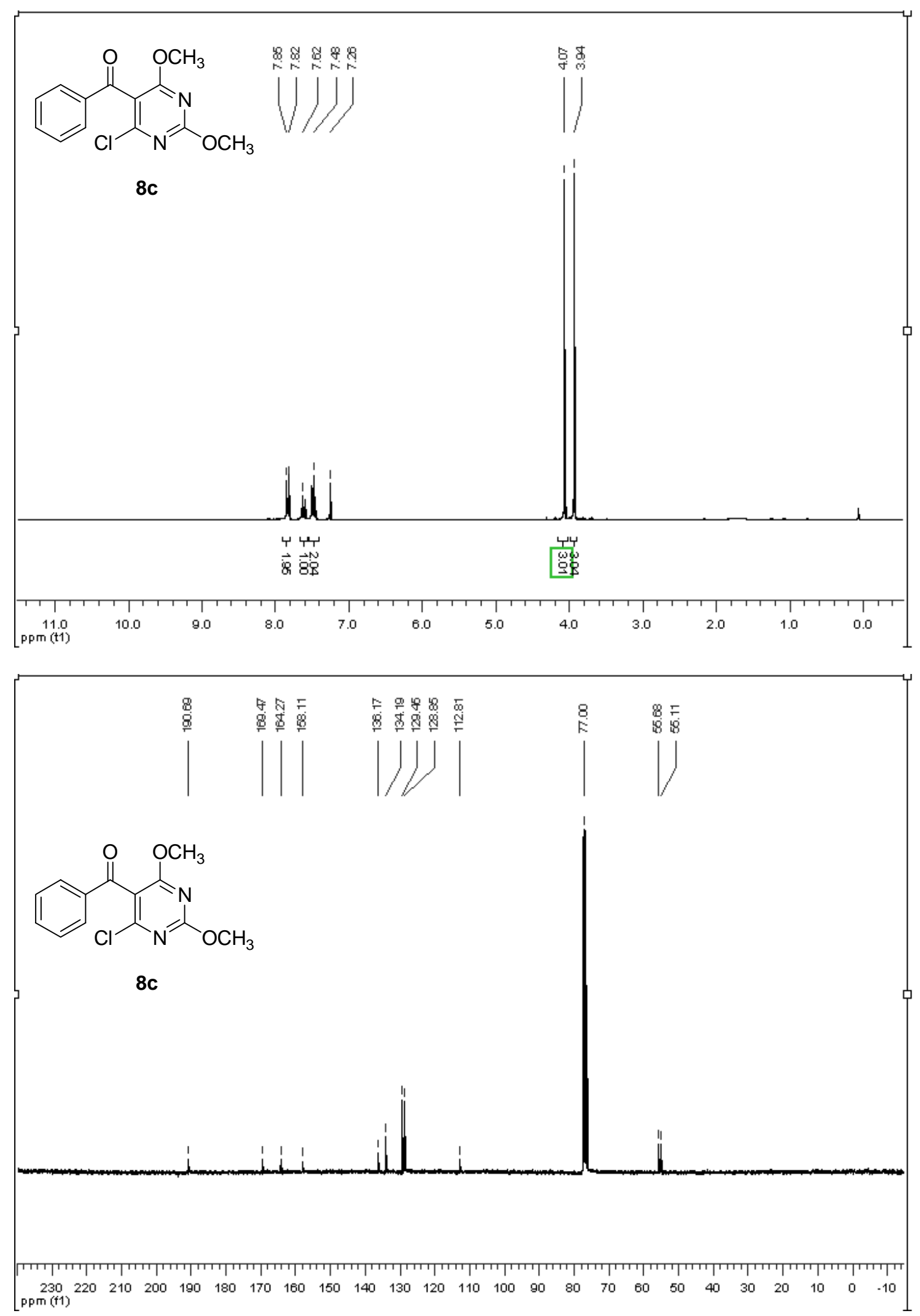

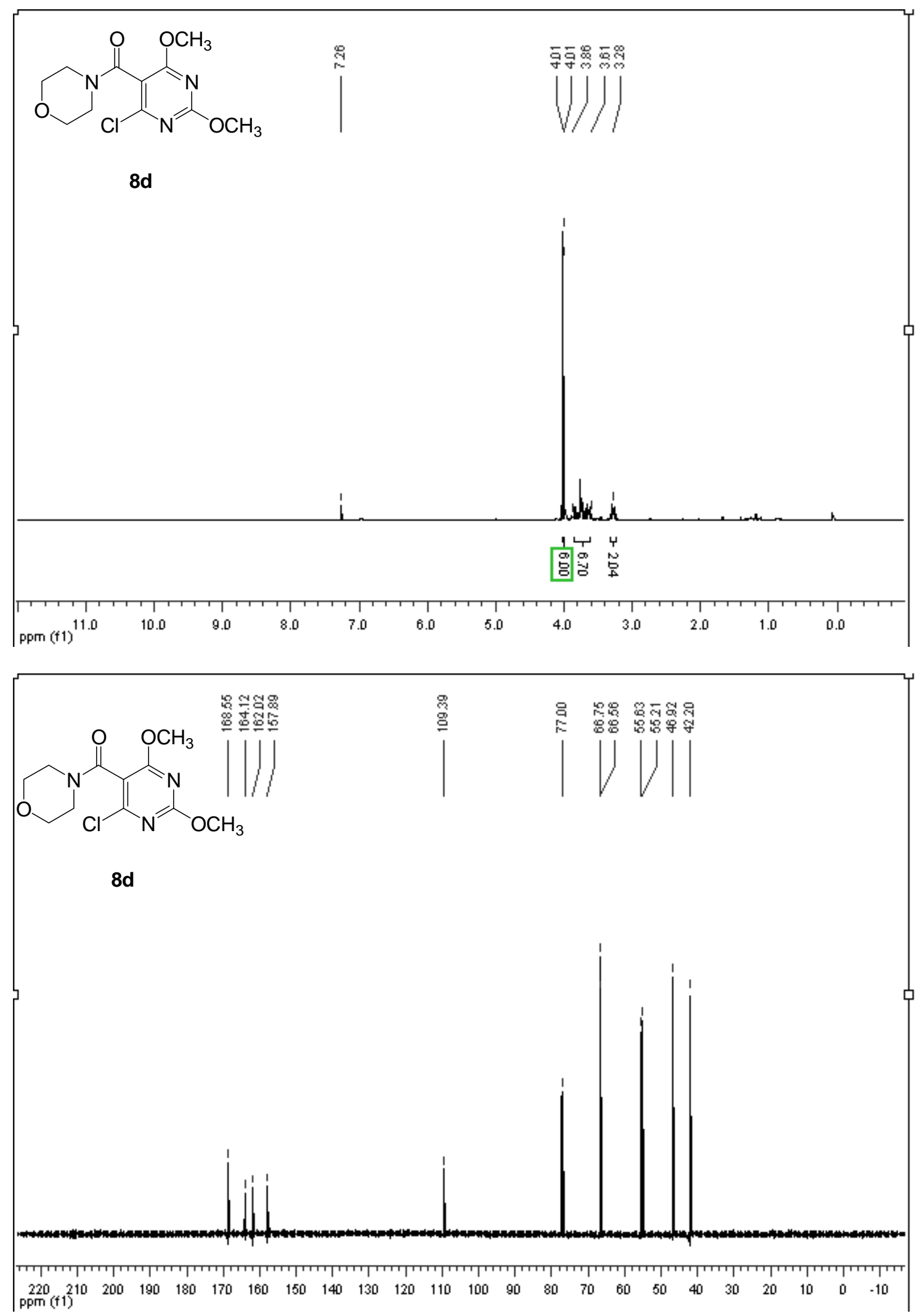

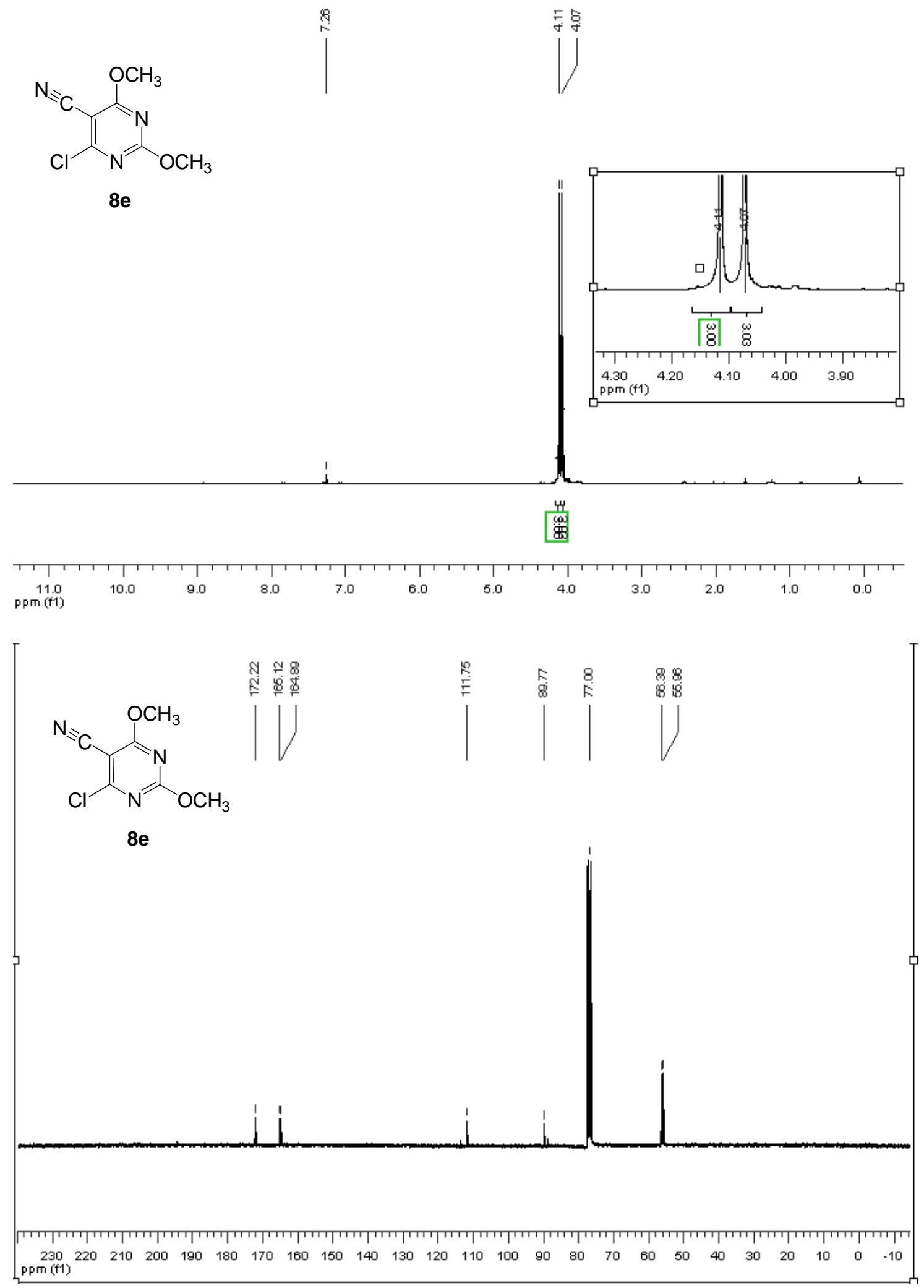

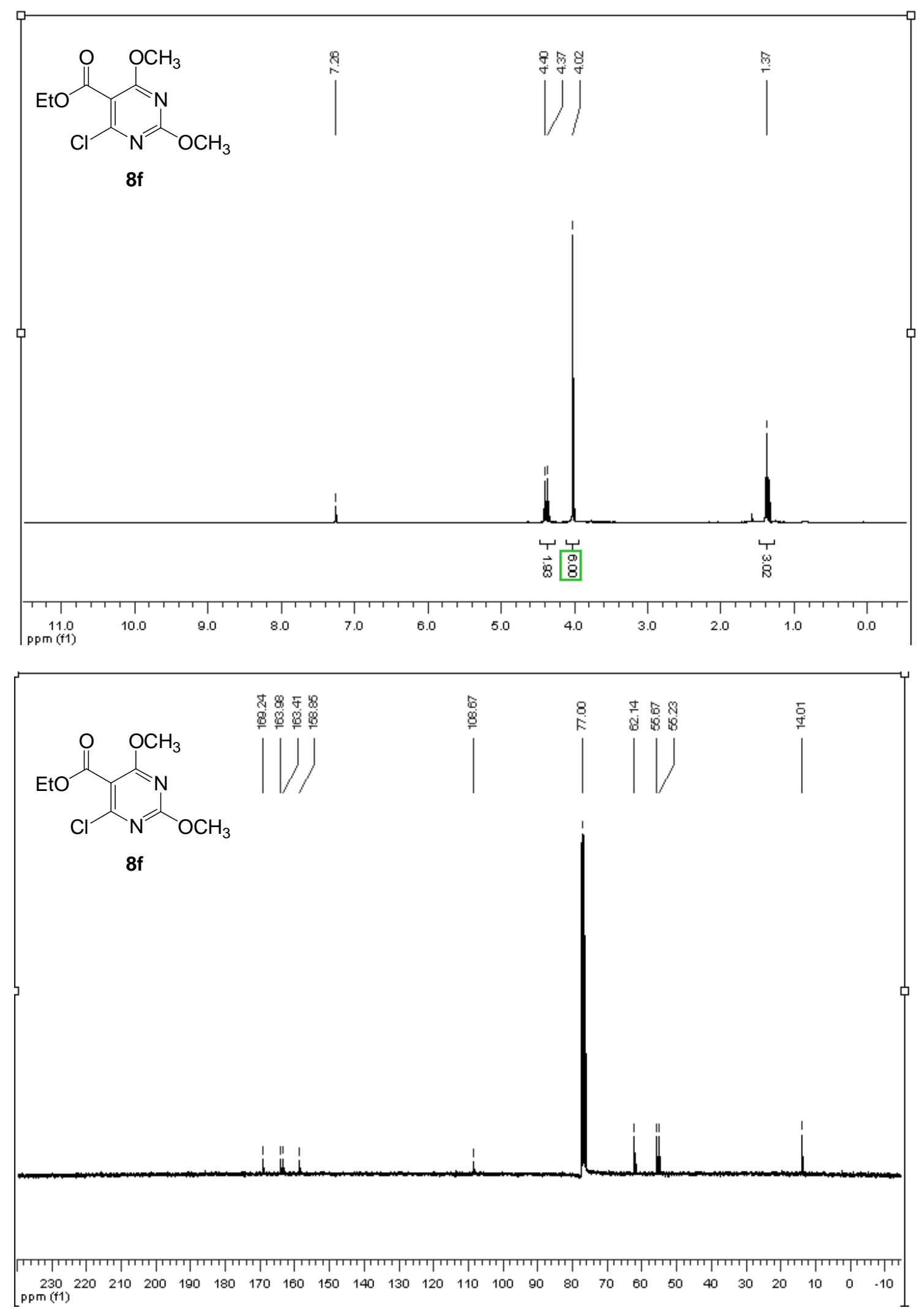

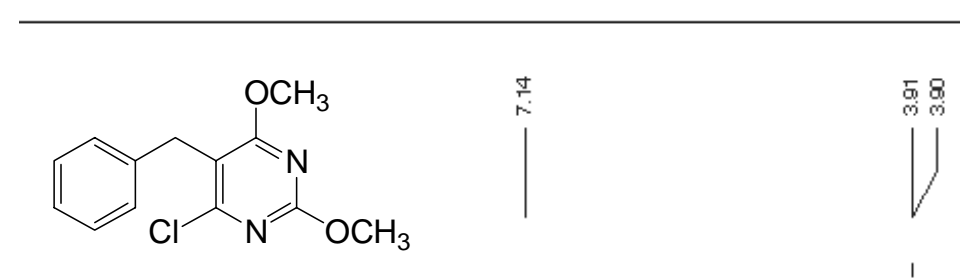

$8 g$
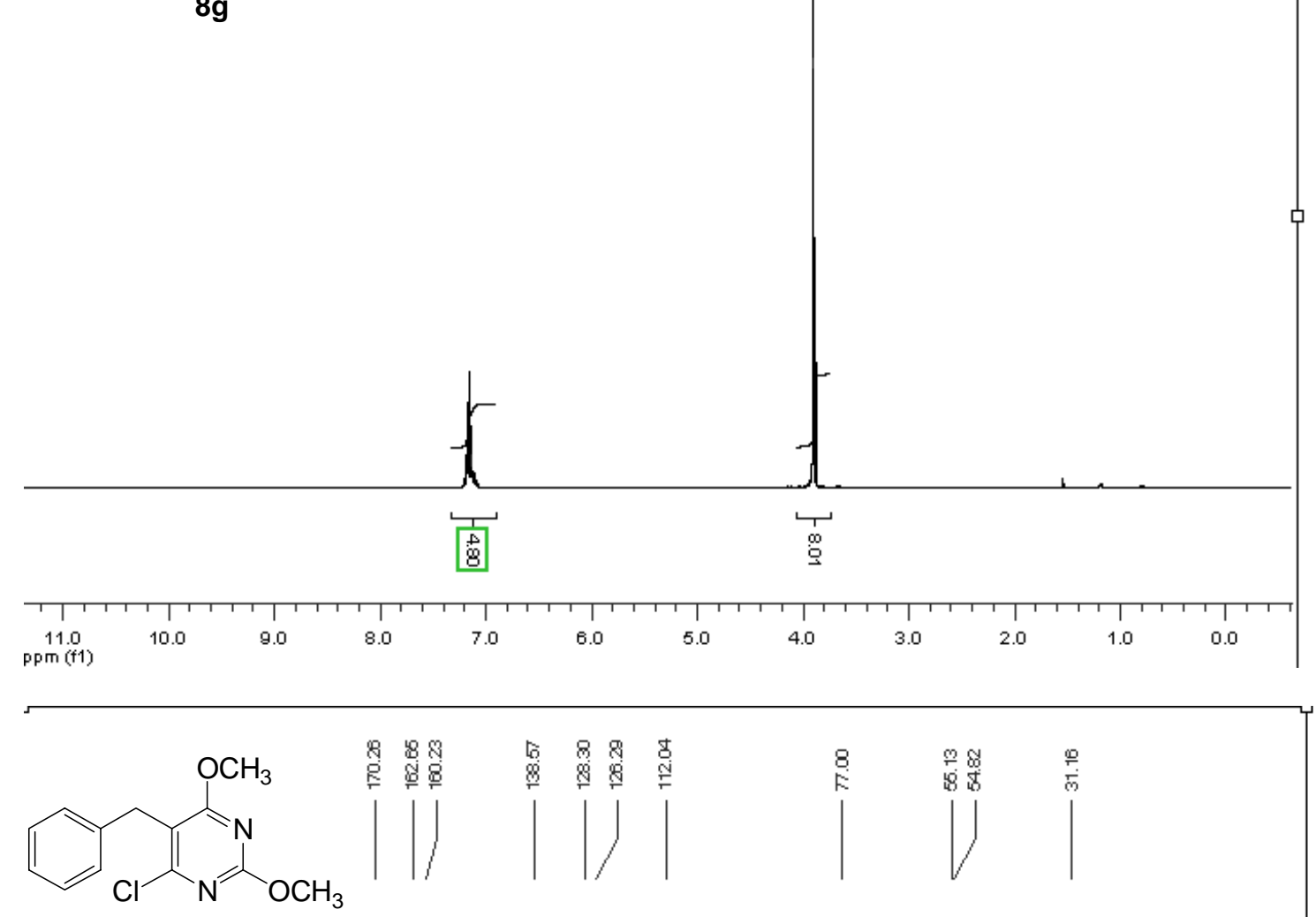

$8 g$

]

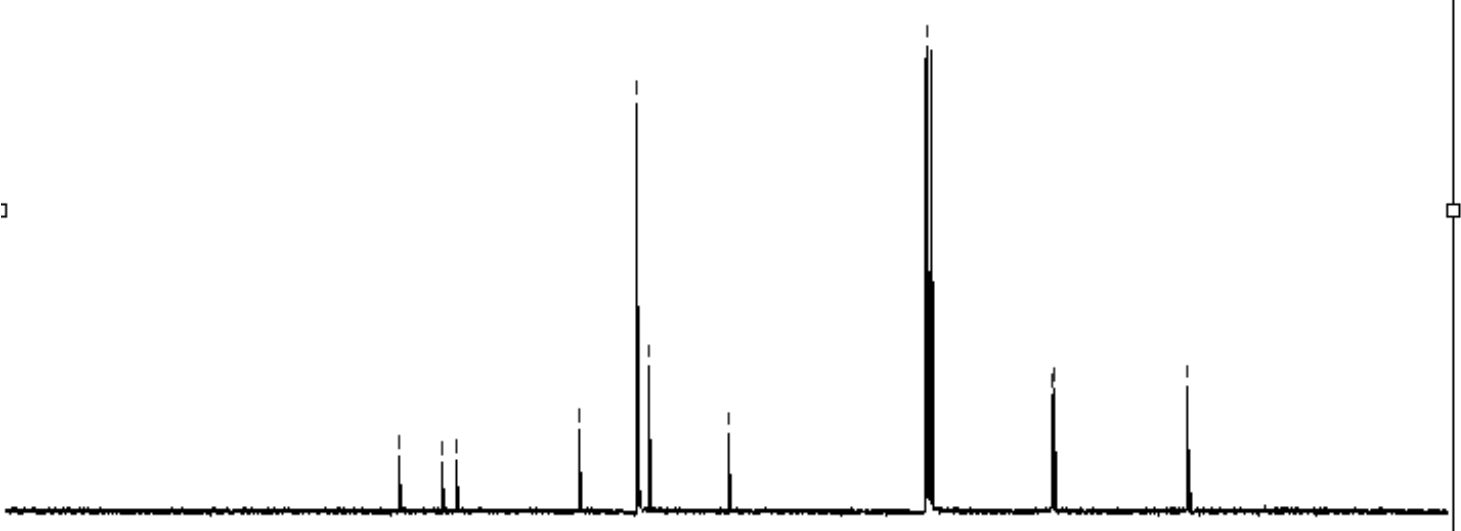

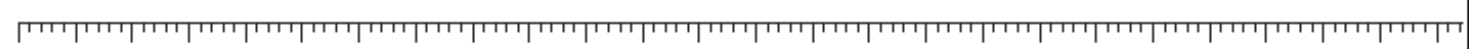

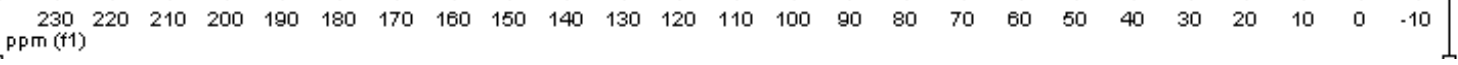



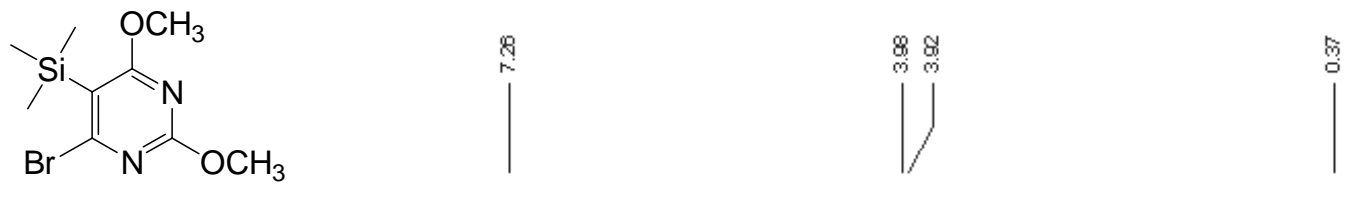

9a

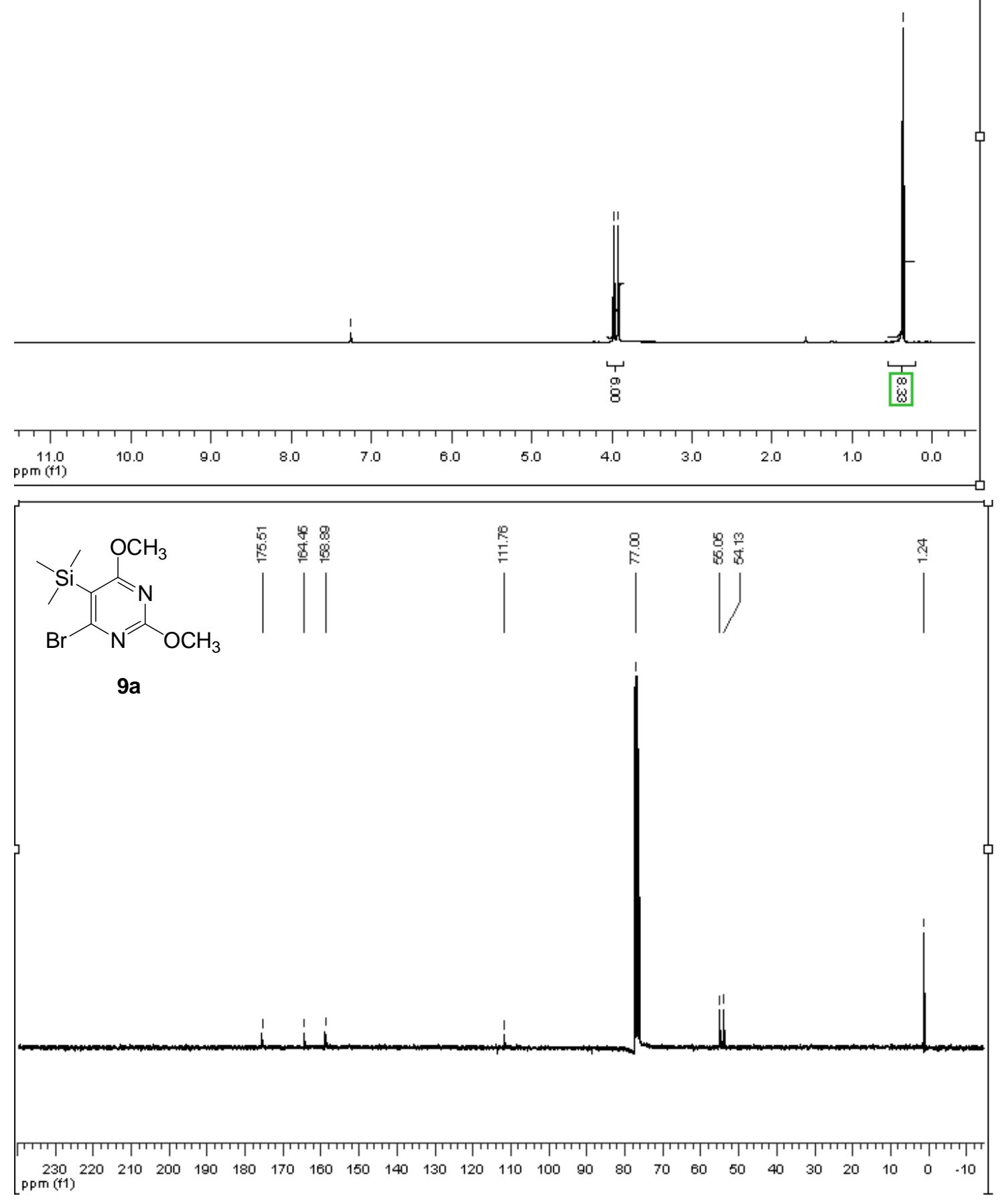




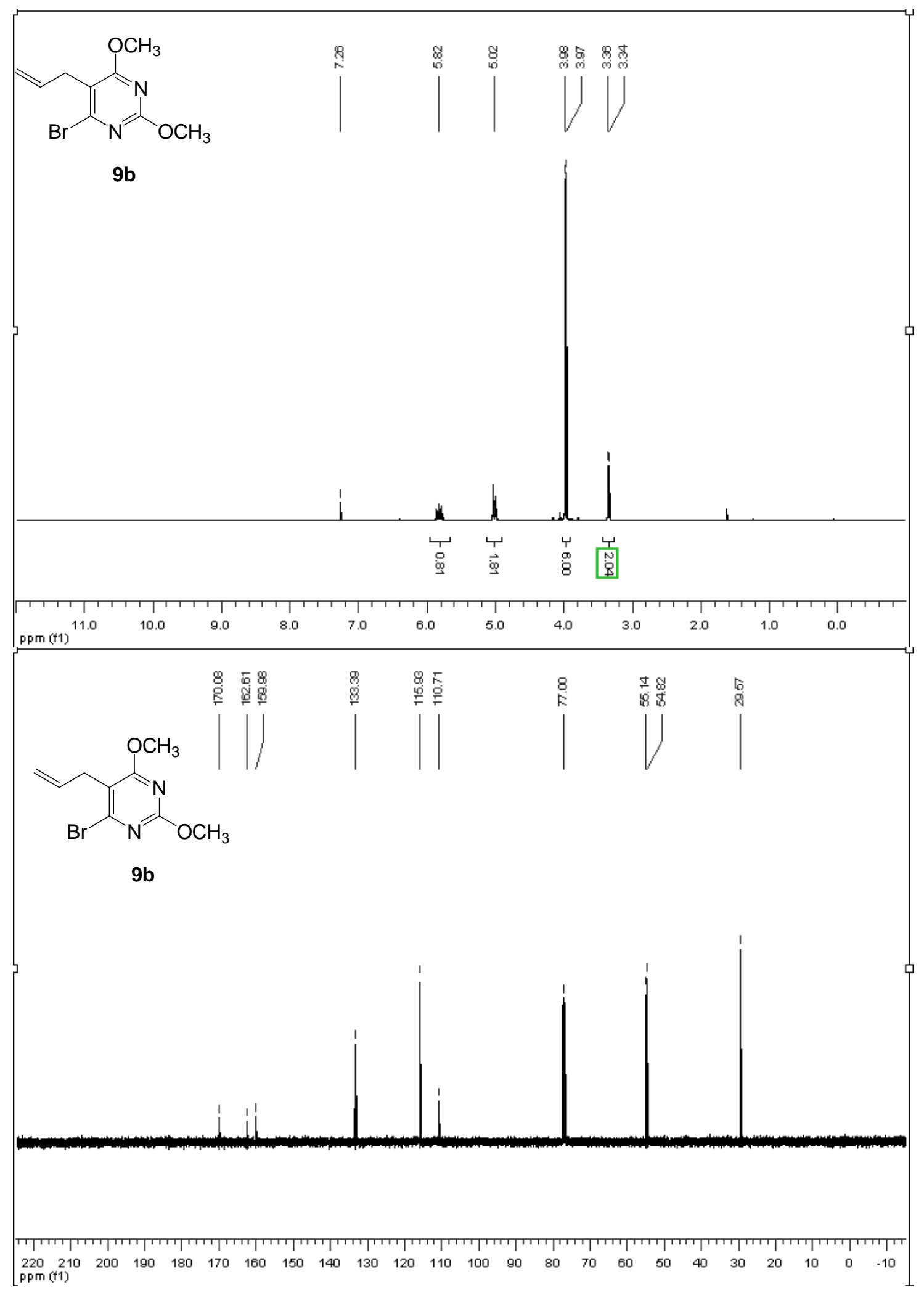



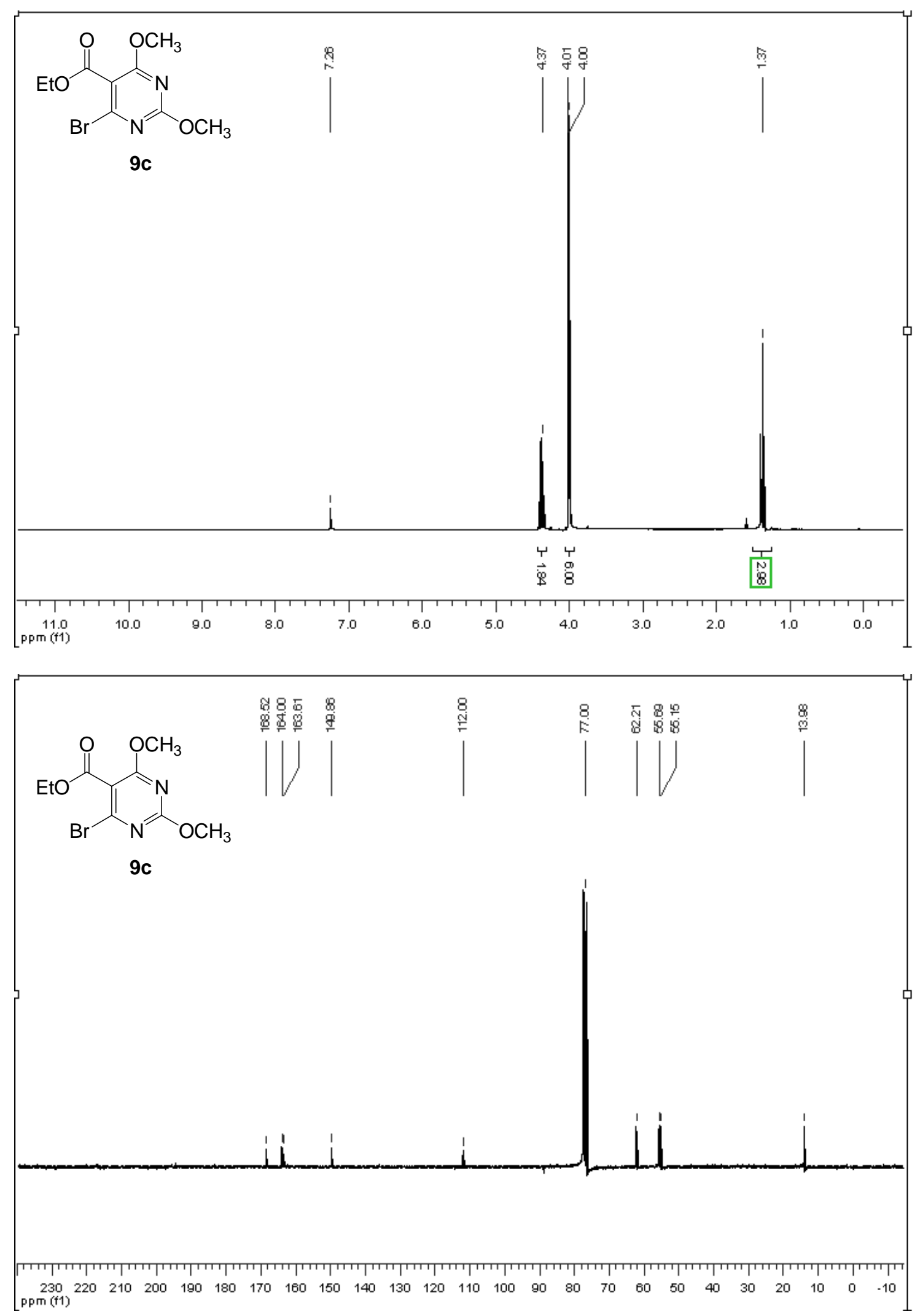

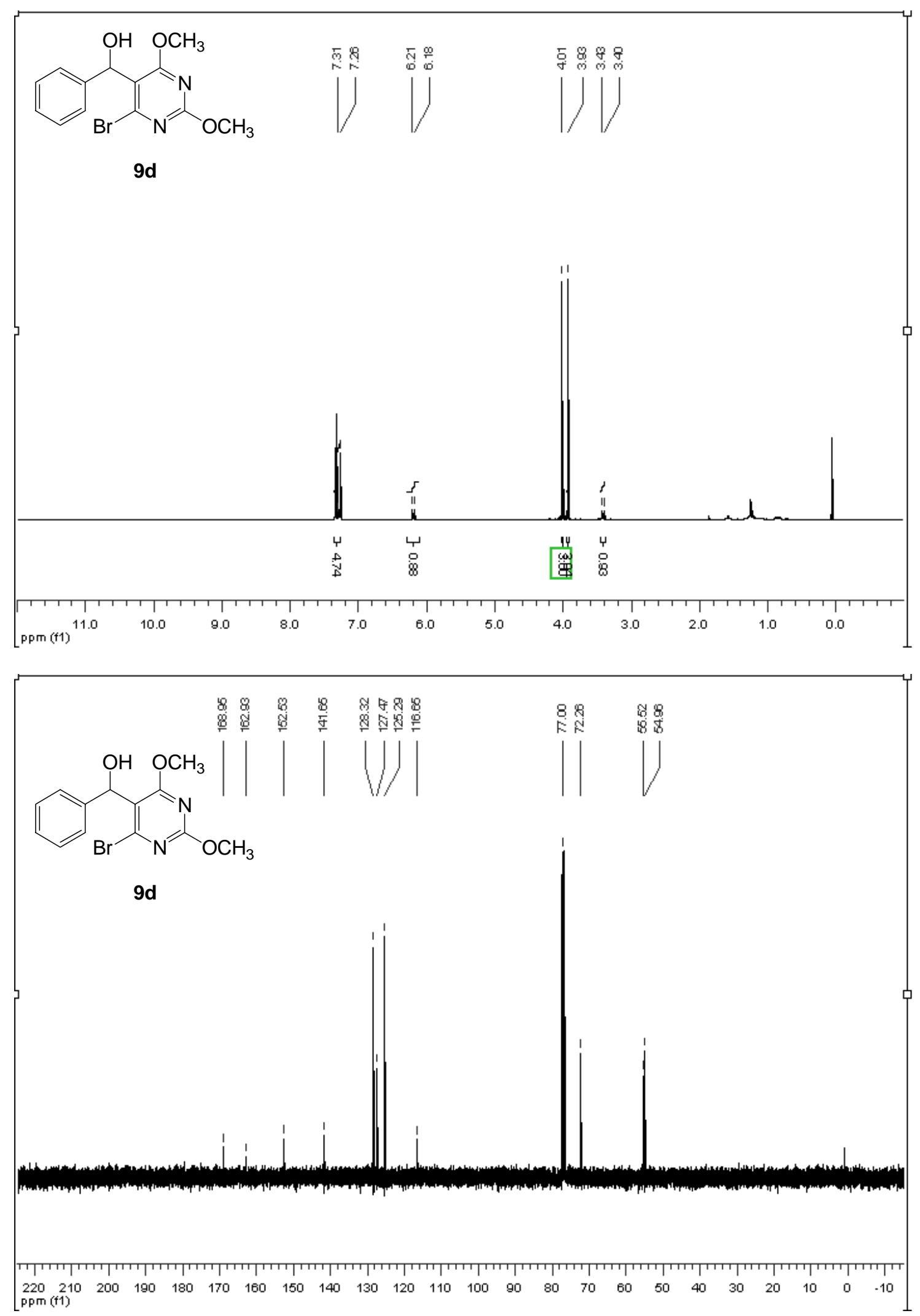


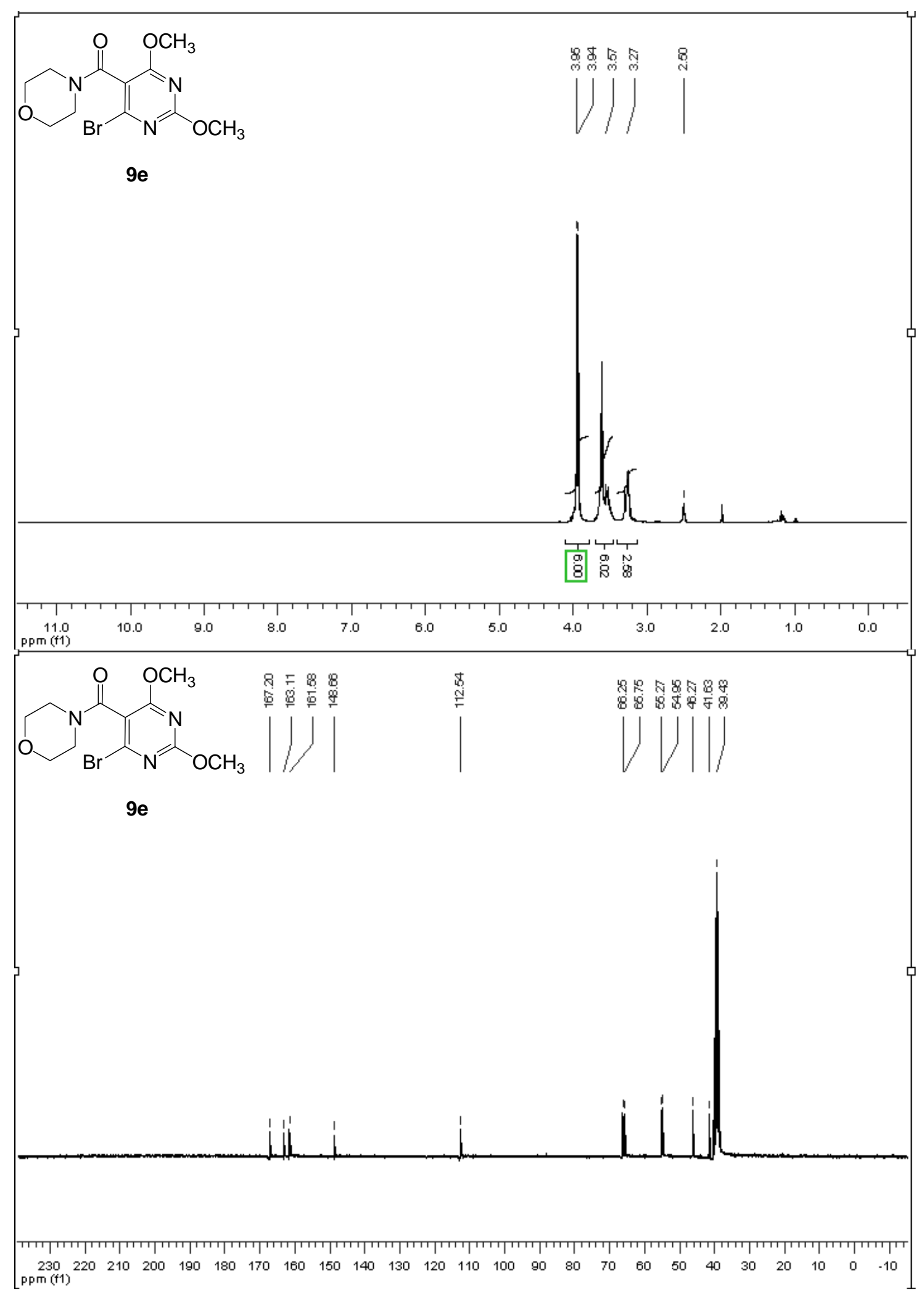



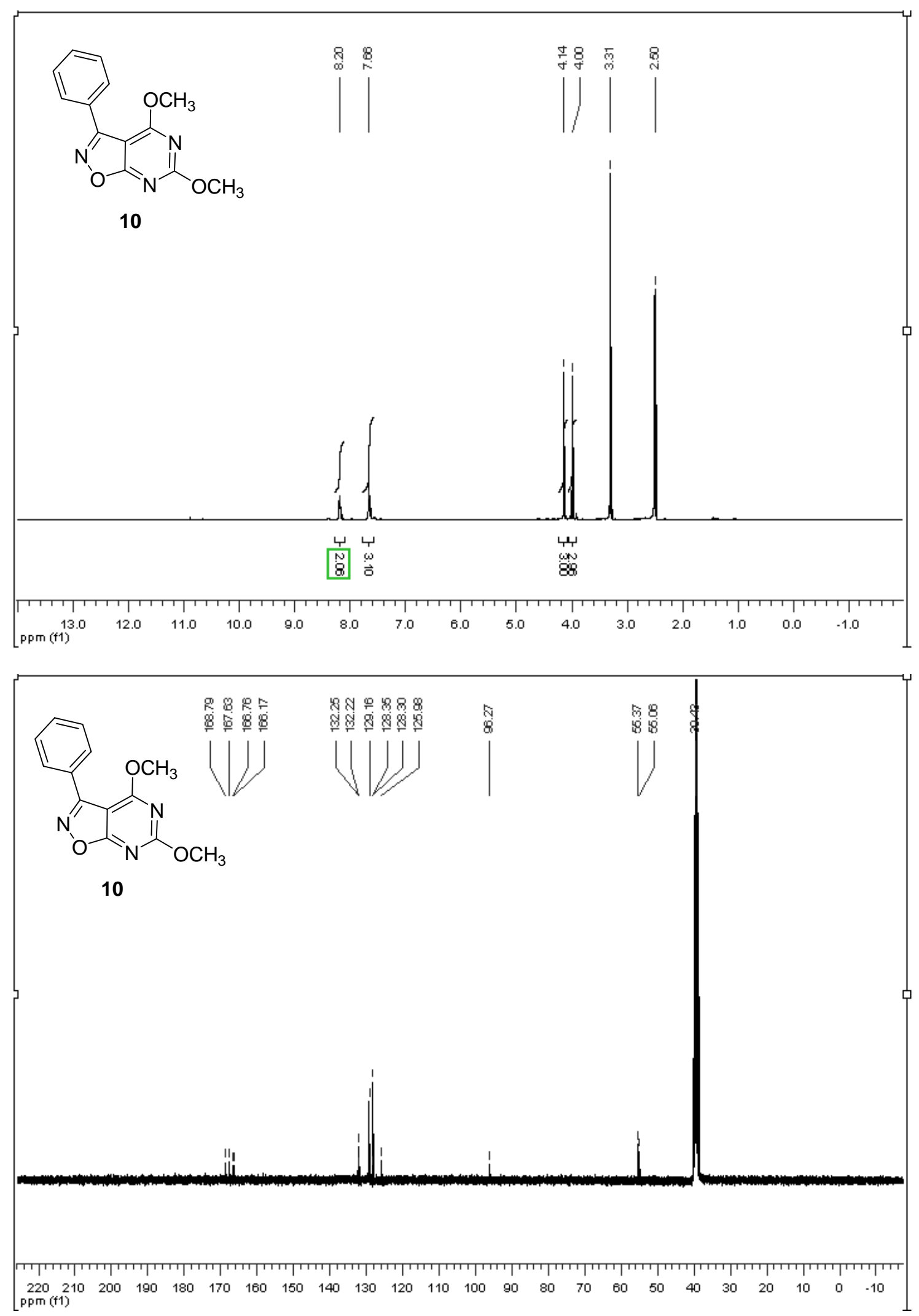

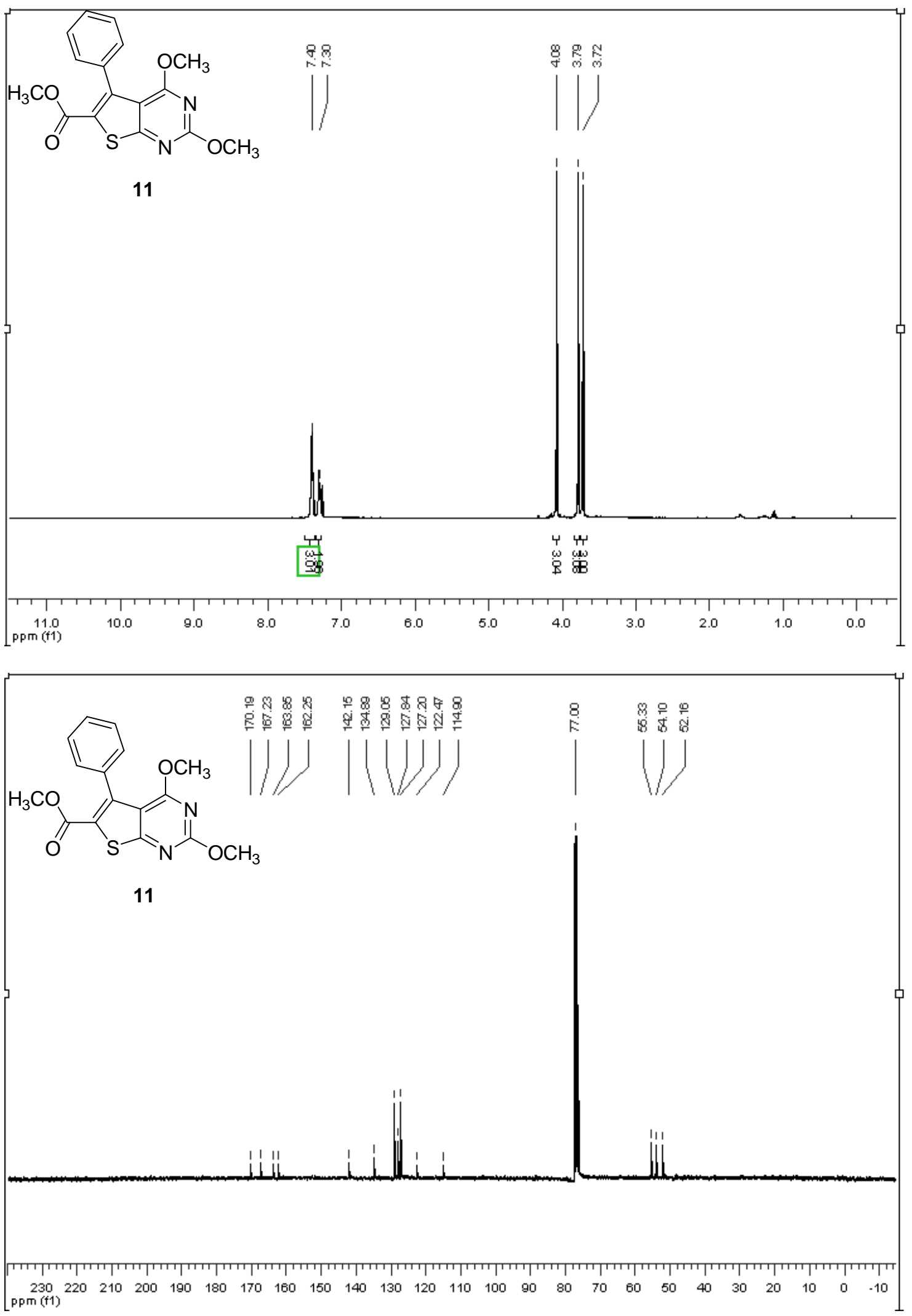

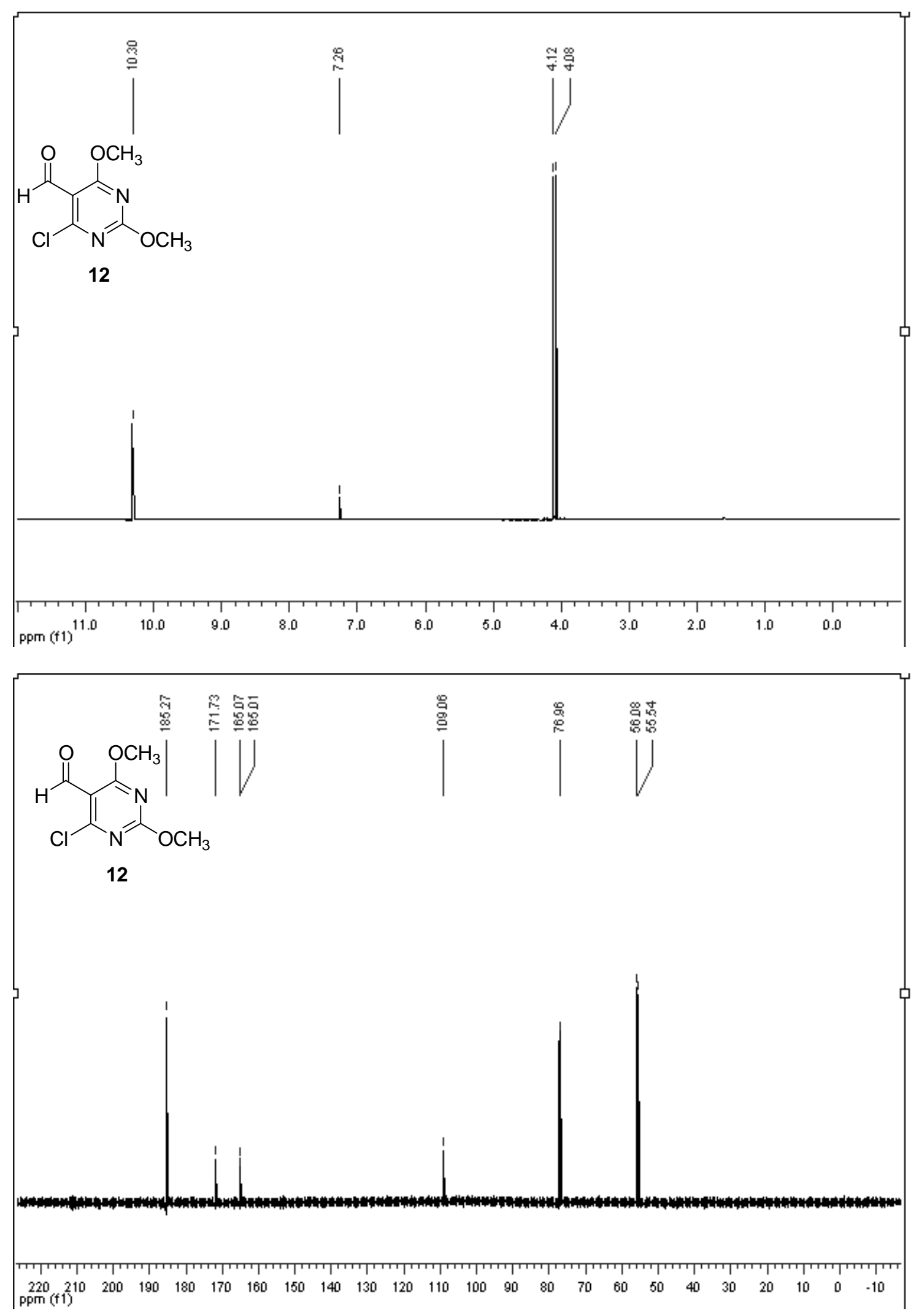

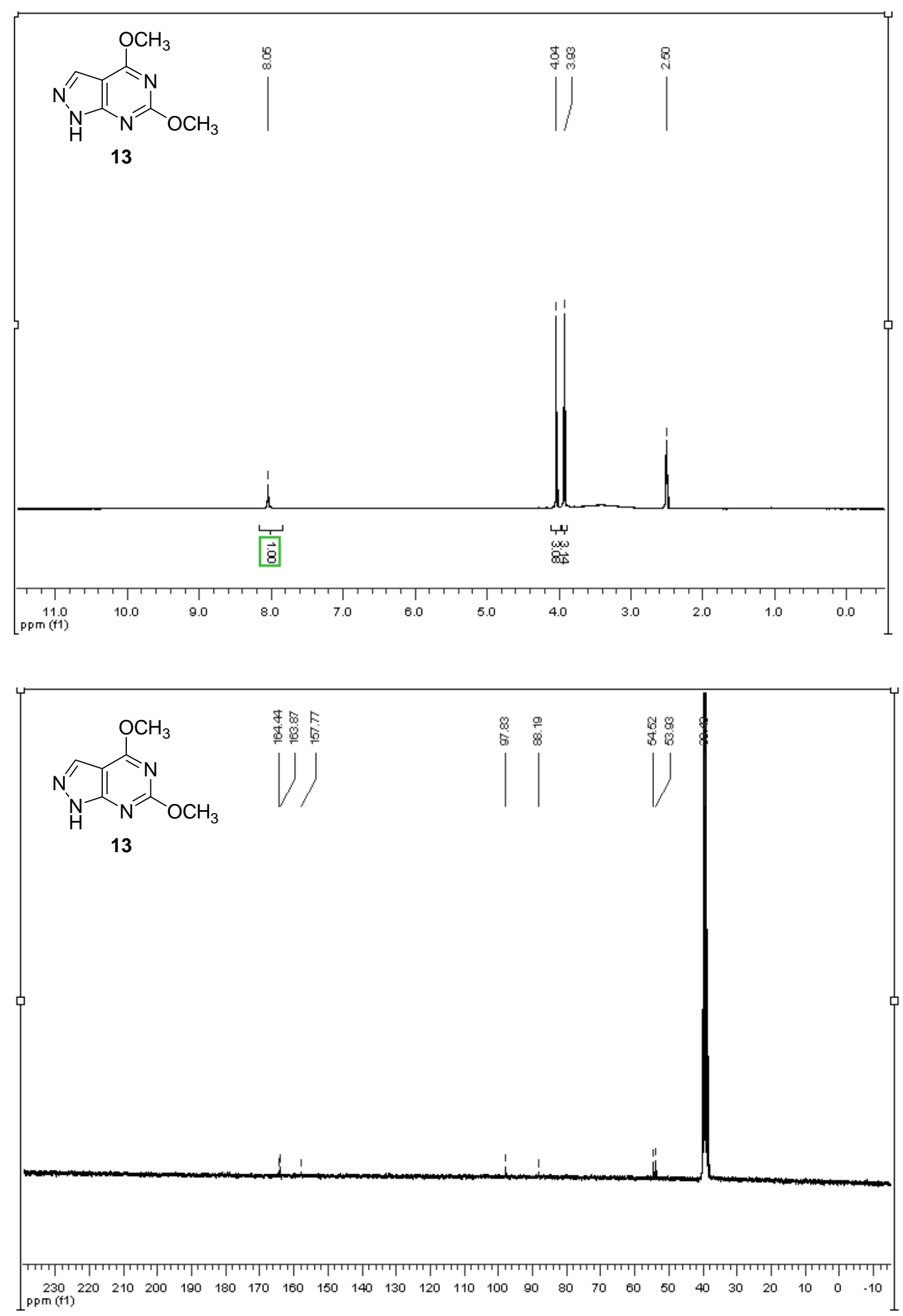

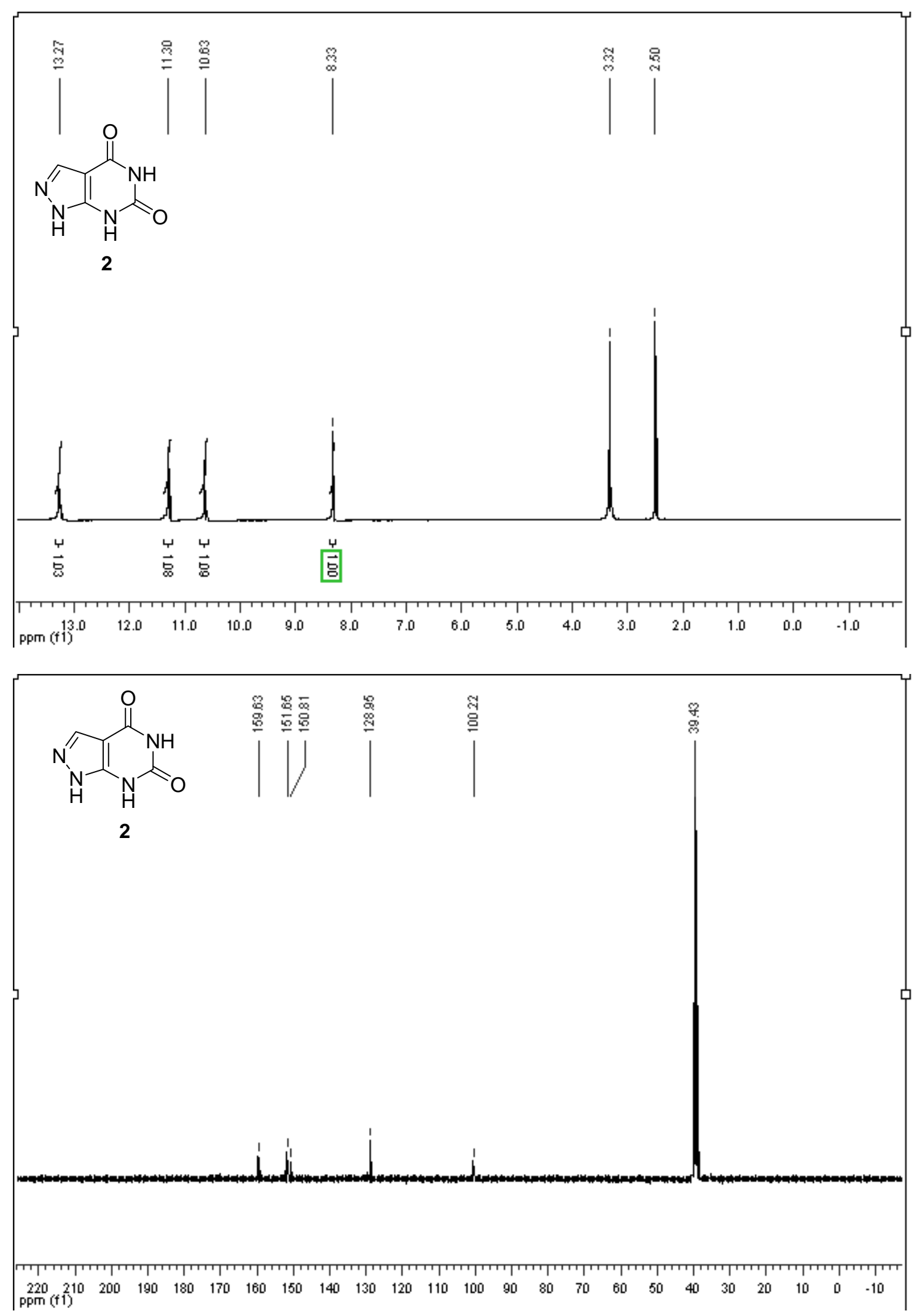

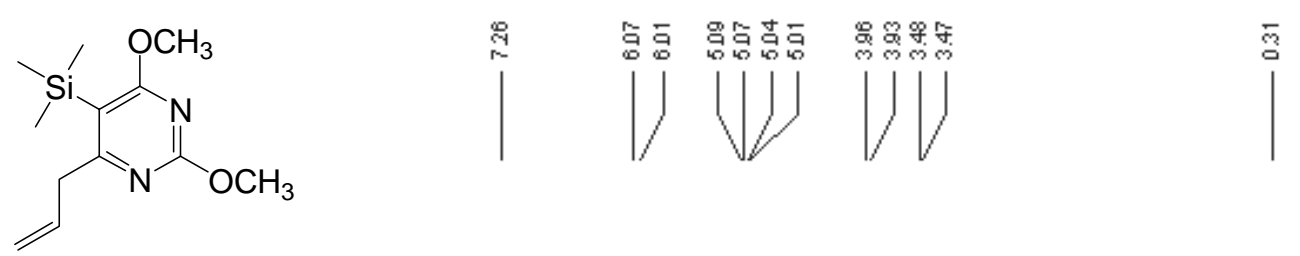

14
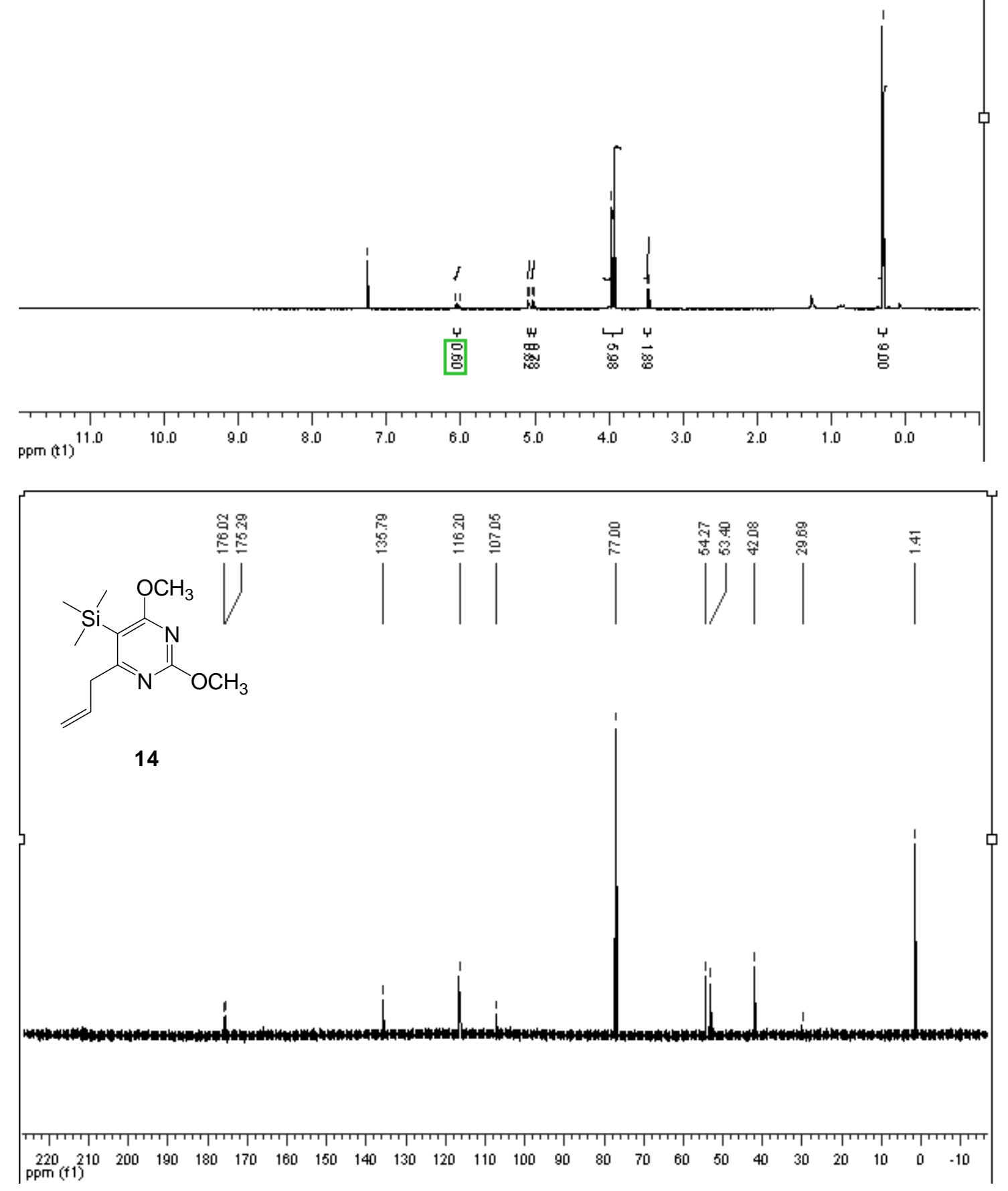

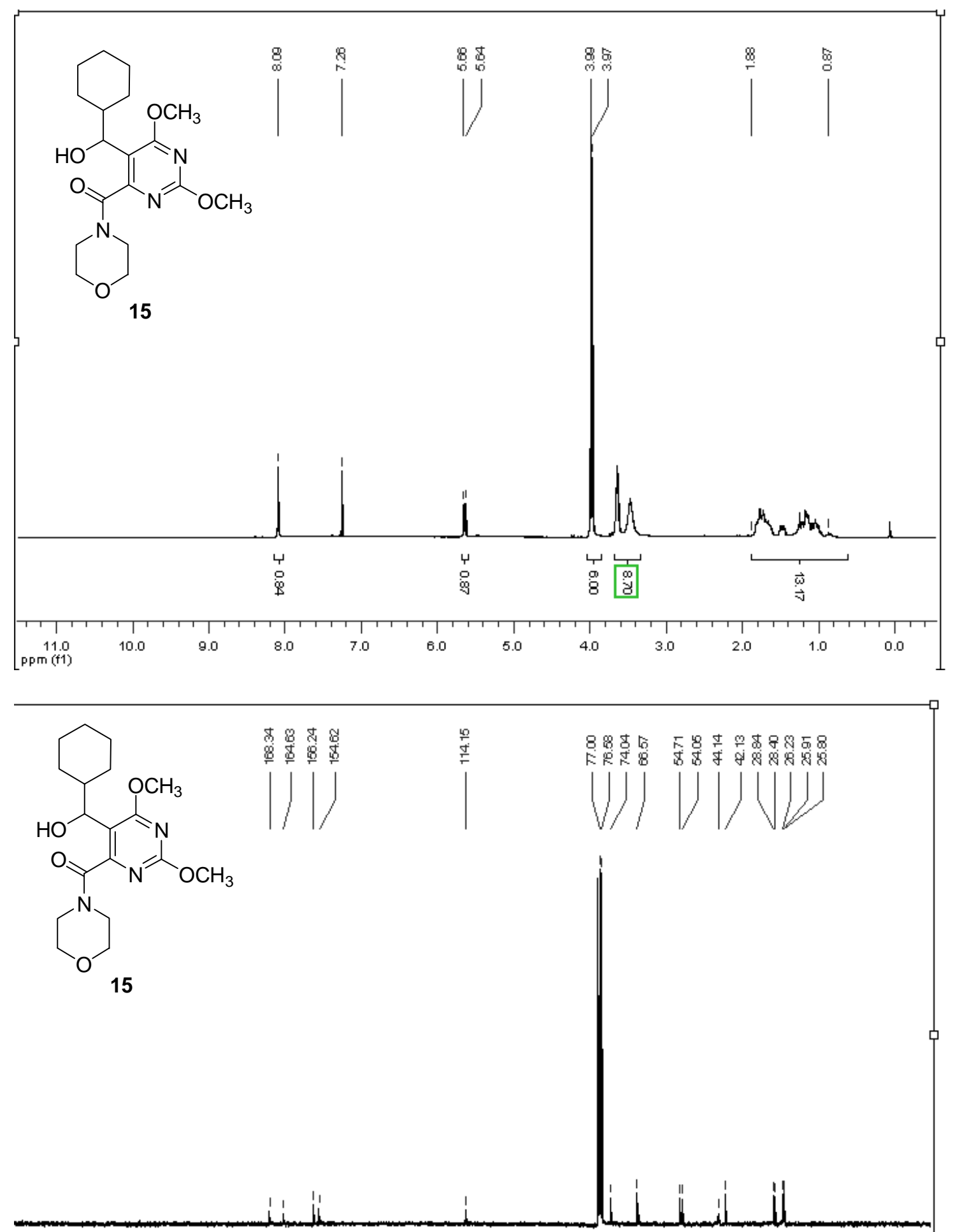

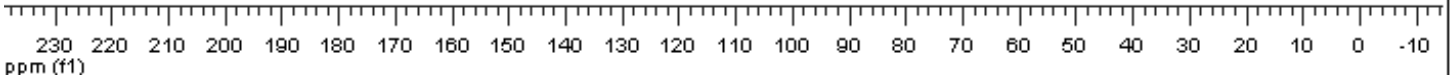




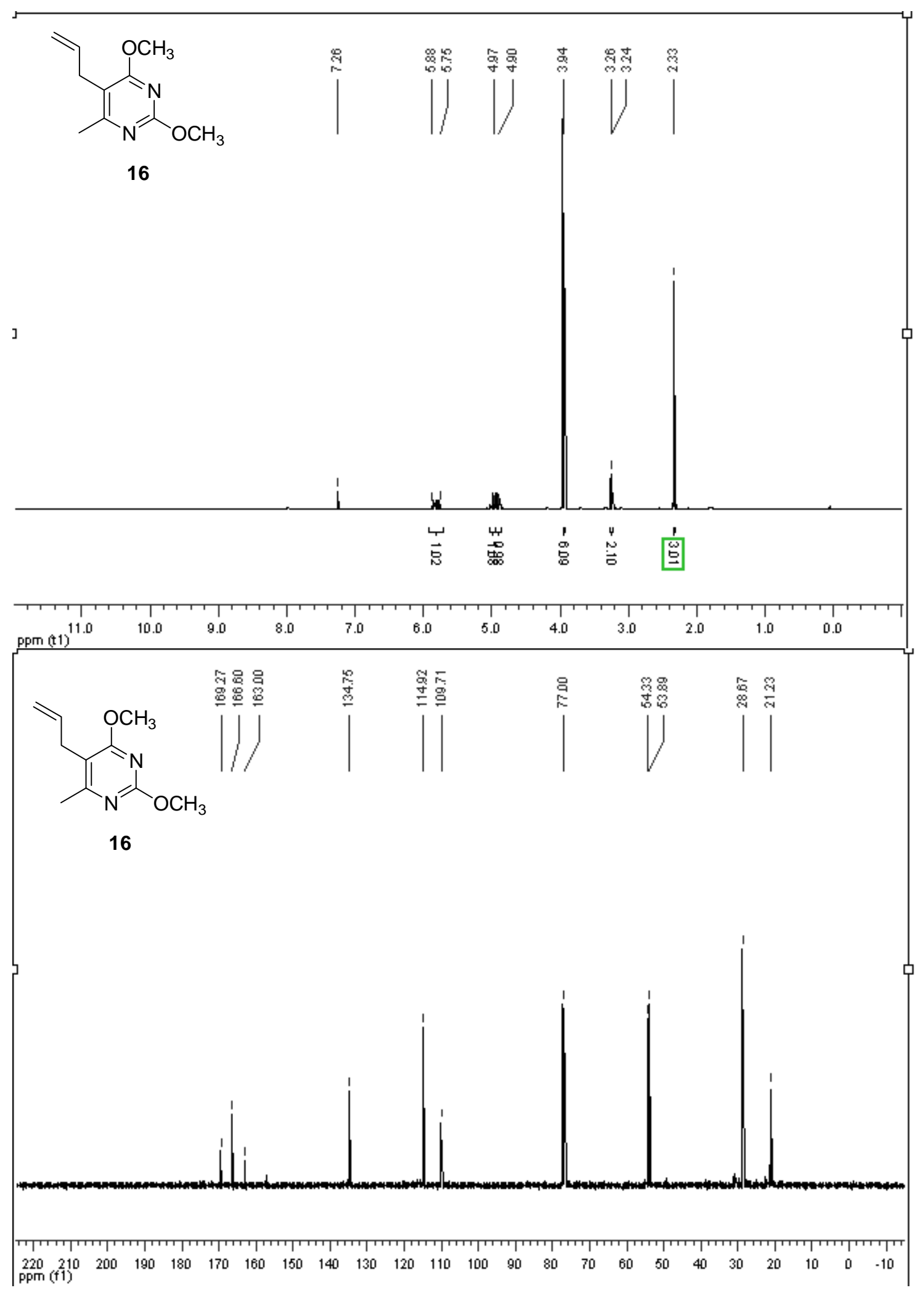



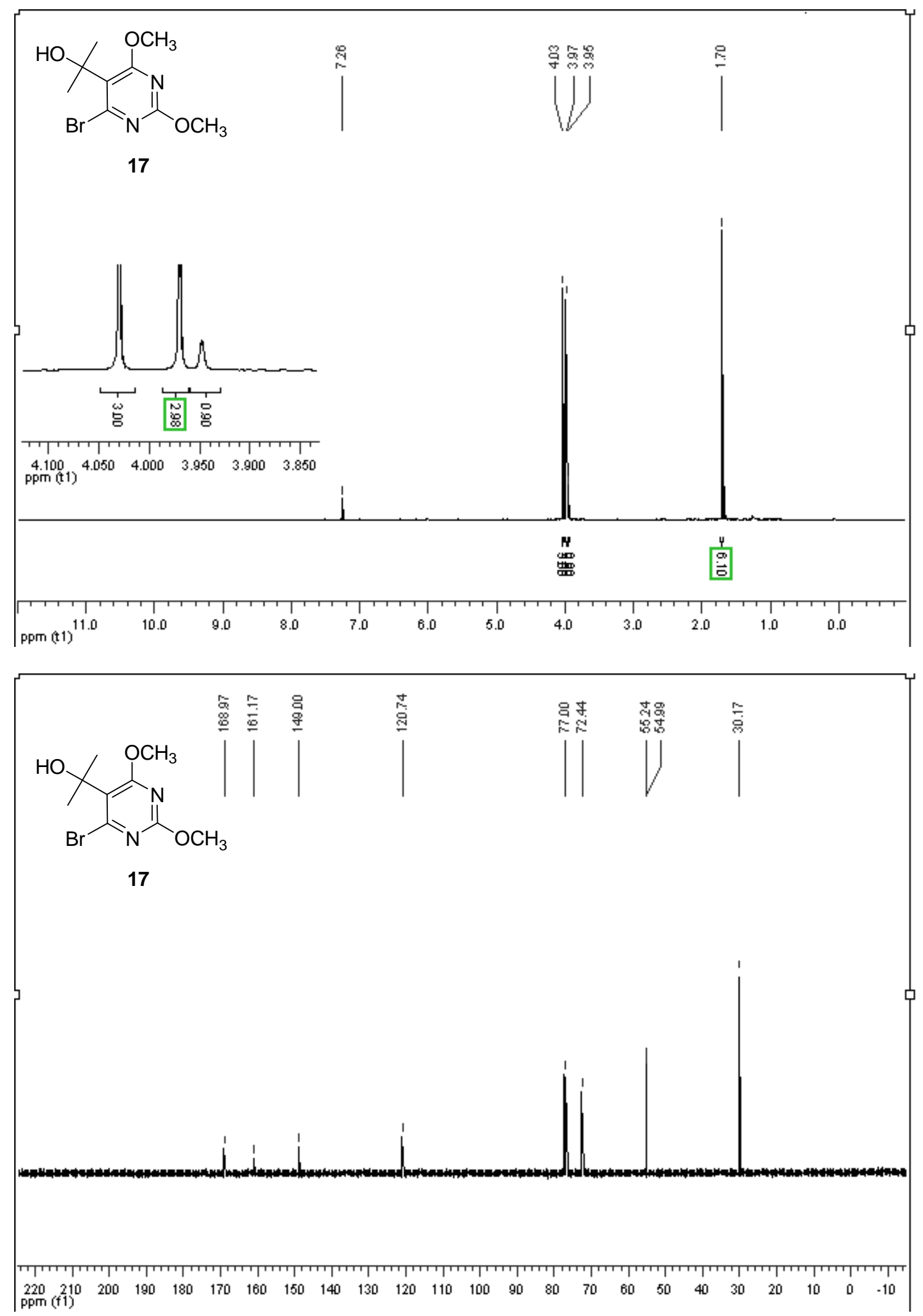

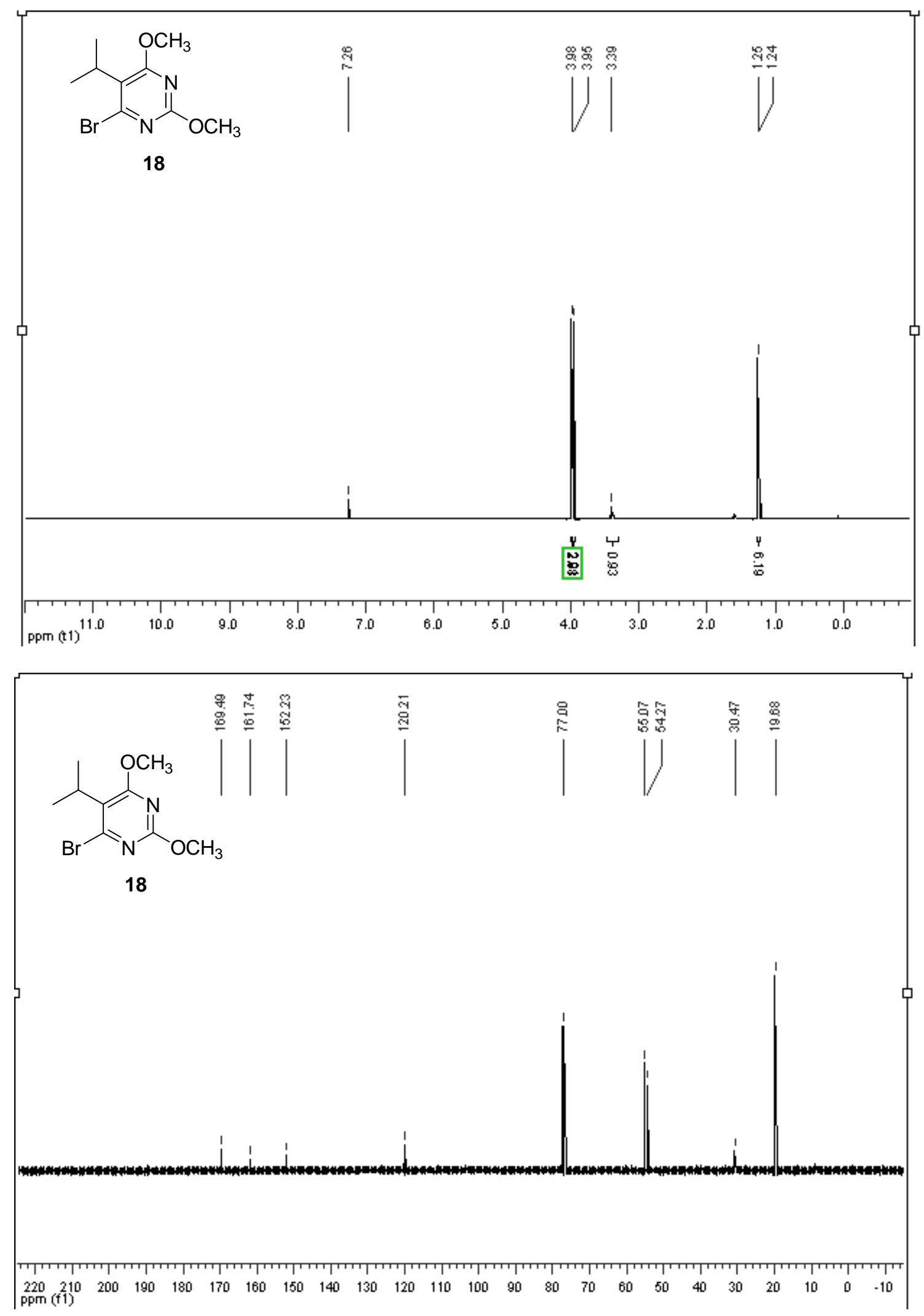

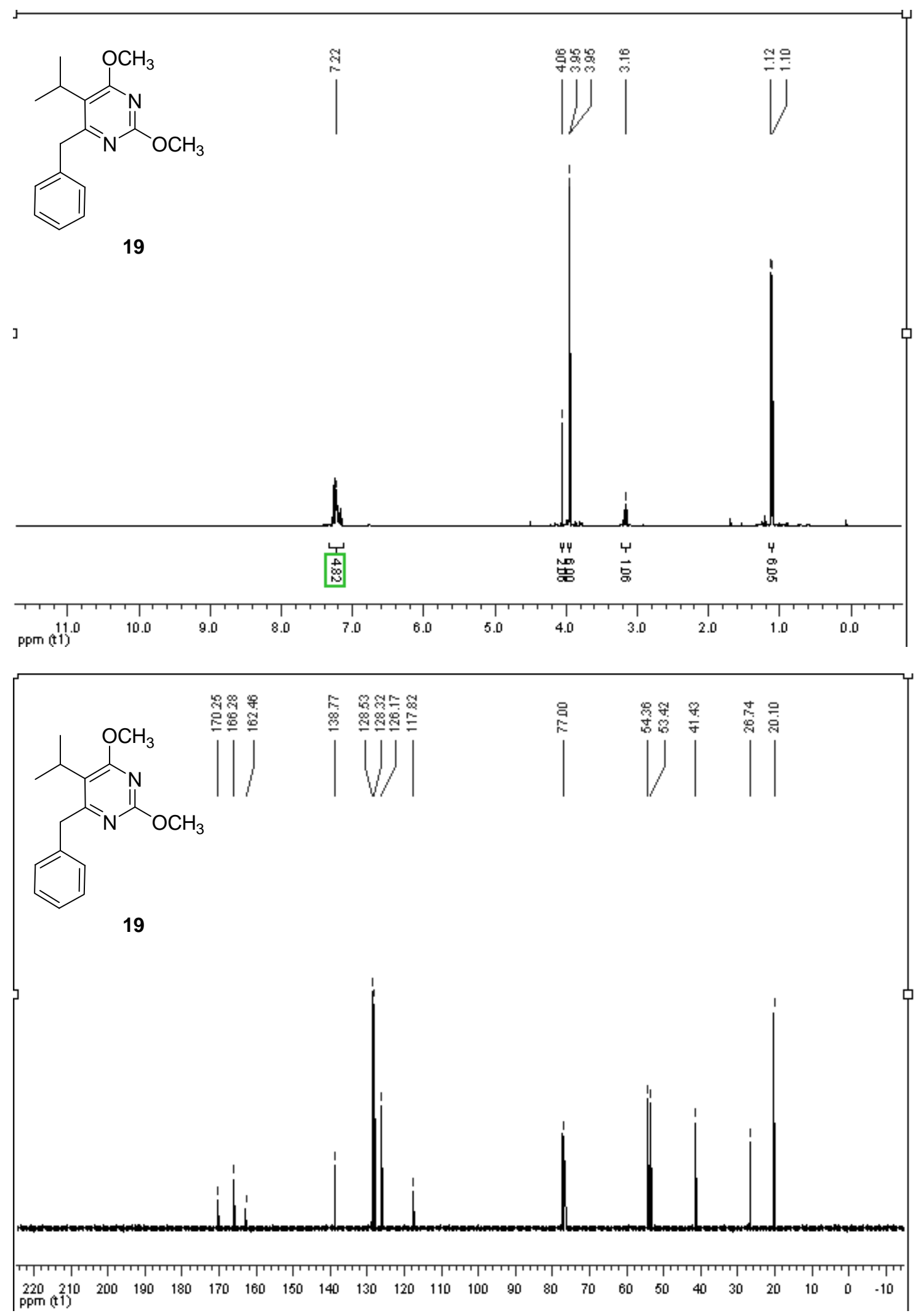

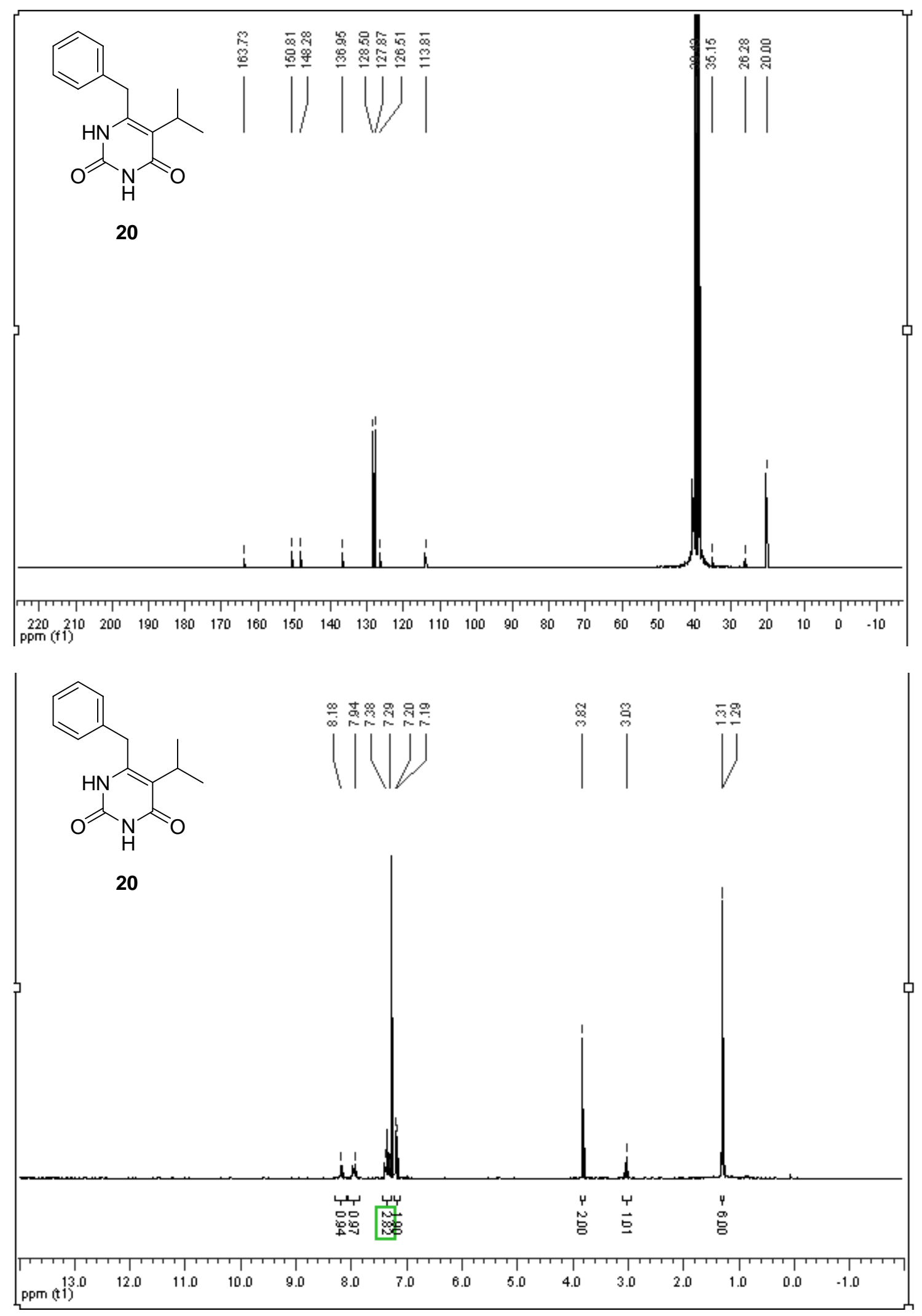

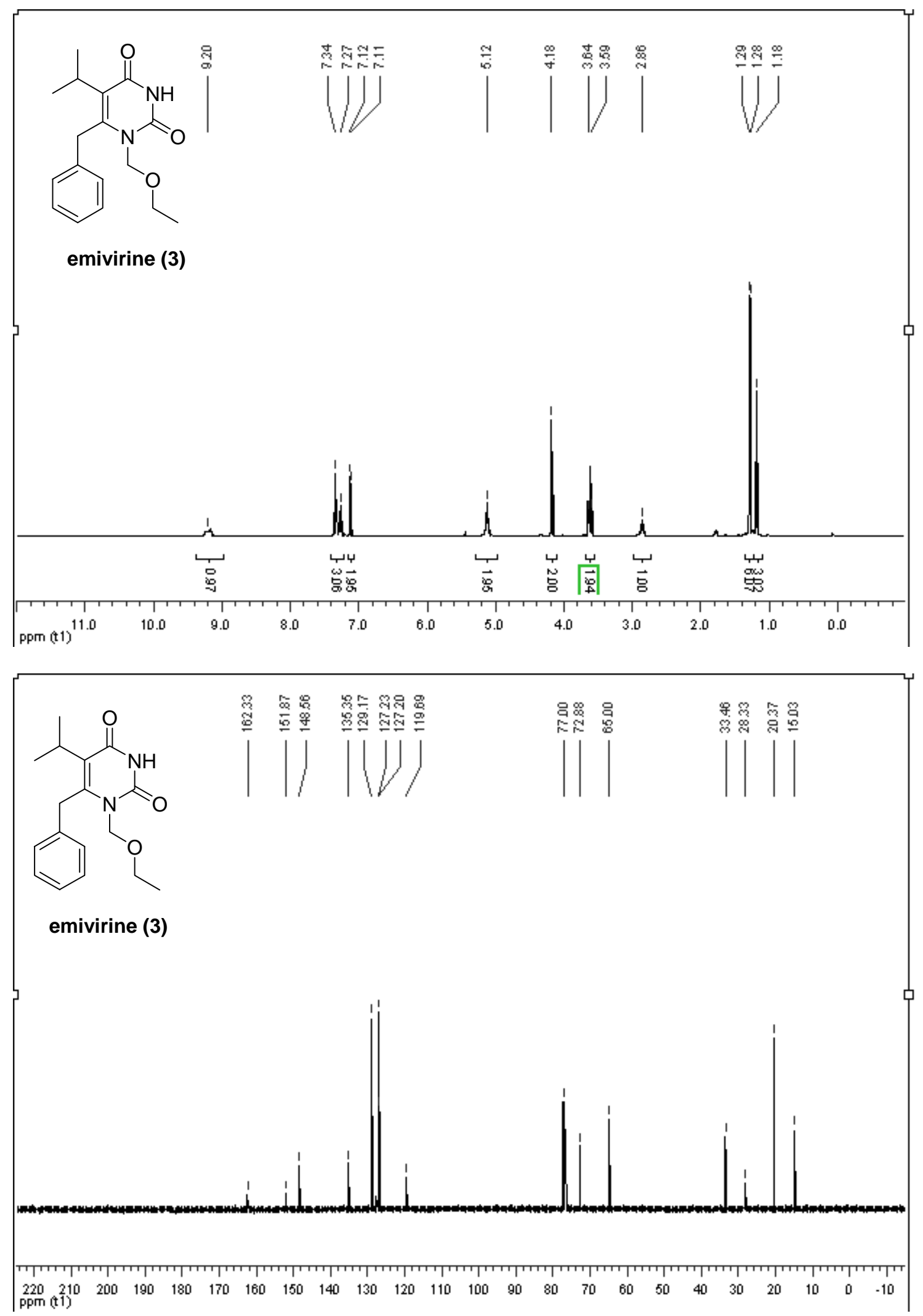